GLASNIK MATEMATIČKI

Vol. 38(58)(2003), $129-155$

\title{
BI-LIPSCHITZ MAPS AND THE CATEGORY OF APPROXIMATE RESOLUTIONS
}

\author{
Takahisa Miyata and Tadashi Watanabe \\ Kobe University and Yamaguchi University, Japan
}

\begin{abstract}
In this paper we consider biLipschitz maps between compact spaces with the metrics which are induced by given approximate resolutions. More precisely, we characterize biLipschitz maps in terms of conditions on any approximate resolutions of the maps. We then show that the box-counting dimension for approximate resolutions which was introduced earlier is invariant under approximate maps corresponding to biLipschitz maps. Moreover, we construct categories whose objects are approximate resolutions and in which the box-counting dimension is invariant.
\end{abstract}

\section{Introduction}

It is well-known that the notion of approximate resolution, which was introduced by Mardešić and Watanabe [5], is useful in many problems in topology $[3,4,13,14,15,12,7]$ and is essential even for compact metric spaces $[2,6,13,14]$. One of the important points in using approximate resolutions is that given a map $f: X \rightarrow Y$ and polyhedral approximate resolutions $\boldsymbol{p}: X \rightarrow \boldsymbol{X}$ and $\boldsymbol{q}: Y \rightarrow \boldsymbol{Y}$ of $X$ and $Y$, respectively, we have an approximate map of systems $\boldsymbol{f}: \boldsymbol{X} \rightarrow \boldsymbol{Y}$ representing $f$.

The authors introduced a new method to study Lipschitz maps, using approximate resolutions in their earlier paper [8]. Given any compact metrizable spaces with an approximate resolution, there is an induced metric that gives the same uniformity, and Lipschitz maps between compact spaces with the so obtained metrics are studied by using approximate resolutions. They also defined and studied the box-counting dimension for approximate resolutions

2000 Mathematics Subject Classification. 54C56, 28A80, 54F45, 54E40.

Key words and phrases. Approximate resolution, biLipschitz map, Lipschitz map, boxcounting dimension, category. 
[9], extending the usual notion of the box-counting dimension for compact subsets in the Euclidean spaces.

The purpose of this paper is to study biLipschitz maps by approximate resolutions and construct categories in which the box-counting dimension is invariant. More specifically, this paper consists of the following two parts: In the first part (Sections 3 and 4), a characterization is given for biLipschitz maps between compact spaces with metrics which are induced by given approximate resolutions, and it is shown that the box-counting dimension is invariant under the approximate maps corresponding to biLipschitz maps. In the second part of the paper (Sections 5 and 6), we construct categories consisting of approximate resolutions and approximate maps corresponding to Lipschitz maps and biLipschitz maps so that the box-counting dimension is invariant in these categories.

Throughout the paper, a space means a compact metric space, and a map means a continuous map unless otherwise stated.

For any space $X$, let $\operatorname{Cov}(X)$ denote the set of all normal open coverings of $X$. For any subset $A$ of $X$ and $\mathcal{U} \in \operatorname{Cov}(X)$, let $\operatorname{st}(A, \mathcal{U})=\cup\{U \in \mathcal{U}$ : $U \cap A \neq \emptyset\}$ and $\mathcal{U} \mid A=\{U \cap A: U \in \mathcal{U}\}$. If $A=\{x\}$, we write $\operatorname{st}(x, \mathcal{U})$ for $\operatorname{st}(\{x\}, \mathcal{U})$. For each $\mathcal{U} \in \operatorname{Cov}(X)$, let $\operatorname{st} \mathcal{U}=\{\operatorname{st}(U, \mathcal{U}): U \in \mathcal{U}\}$. Let $\mathrm{st}^{n+1} \mathcal{U}=\mathrm{st}\left(\mathrm{st}^{n} \mathcal{U}\right)$ for each $n=1,2, \ldots$ and $\mathrm{st}^{1} \mathcal{U}=\mathrm{st} \mathcal{U}$. For any metric space $(X, \mathrm{~d})$ and $r>0$, let $\mathrm{U}_{\mathrm{d}}(x, r)=\{y \in X: \mathrm{d}(x, y)<r\}$. For any $\mathcal{U} \in \operatorname{Cov}(X)$, two points $x, x^{\prime} \in X$ are $\mathcal{U}$-near, denoted $\left(x, x^{\prime}\right)<\mathcal{U}$, provided $x, x^{\prime} \in U$ for some $U \in \mathcal{U}$. For any $\mathcal{V} \in \operatorname{Cov}(Y)$, two maps $f, g: X \rightarrow Y$ between spaces are $\mathcal{V}$-near, denoted $(f, g)<\mathcal{V}$, provided $(f(x), g(x))<\mathcal{V}$ for each $x \in X$. For each $\mathcal{U} \in \operatorname{Cov}(X)$ and $\mathcal{V} \in \operatorname{Cov}(Y)$, let $f \mathcal{U}=\{f(U): U \in \mathcal{U}\}$ and $f^{-1} \mathcal{V}=\left\{f^{-1}(V): V \in \mathcal{V}\right\}$. Let $\mathbb{N}$ denote the set of natural numbers with the usual order.

\section{Approximate Resolutions And IndUCED METRICS}

In this section we recall the definitions and properties of approximate resolutions and the results concerning Lipschitz maps which will be needed in later sections. For more details on approximate resolutions and Lipschitz maps, the reader is referred to $[5]$ and $[8,9]$, respectively.

An approximate inverse sequence (approximate sequence, in short) $\boldsymbol{X}=$ $\left\{X_{i}, \mathcal{U}_{i}, p_{i i^{\prime}}\right\}$ consists of

i) a sequence of spaces $X_{i}, i \in \mathbb{N}$;

ii) a sequence of $\mathcal{U}_{i} \in \operatorname{Cov}\left(X_{i}\right), i \in \mathbb{N}$; and

iii) maps $p_{i i^{\prime}}: X_{i^{\prime}} \rightarrow X_{i}$ for $i<i^{\prime}$ where $p_{i i}=1_{X_{i}}$ the identity map on $X_{i}$.

It must satisfy the following three conditions:

(A1) $\left(p_{i i^{\prime}} p_{i^{\prime} i^{\prime \prime}}, p_{i i^{\prime \prime}}\right)<\mathcal{U}_{i}$ for $i<i^{\prime}<i^{\prime \prime}$; 
(A2) For each $i \in \mathbb{N}$ and $\mathcal{U} \in \operatorname{Cov}\left(X_{i}\right)$, there exists $i^{\prime}>i$ such that $\left(p_{i i_{1}} p_{i_{1} i_{2}}, p_{i i_{2}}\right)<\mathcal{U}$ for $i^{\prime}<i_{1}<i_{2}$; and

(A3) For each $i \in \mathbb{N}$ and $\mathcal{U} \in \operatorname{Cov}\left(X_{i}\right)$, there exists $i^{\prime}>i$ such that $\mathcal{U}_{i^{\prime \prime}}<p_{i i^{\prime \prime}}^{-1} \mathcal{U}$ for $i^{\prime}<i^{\prime \prime}$.

An approximate map $\boldsymbol{p}=\left\{p_{i}\right\}: X \rightarrow \boldsymbol{X}$ of a space $X$ into an approximate sequence $\boldsymbol{X}=\left\{X_{i}, \mathcal{U}_{i}, p_{i i^{\prime}}\right\}$ consists of maps $p_{i}: X \rightarrow X_{i}$ for $i \in \mathbb{N}$ with the following property:

(AS) For each $i \in \mathbb{N}$ and $\mathcal{U} \in \operatorname{Cov}\left(X_{i}\right)$, there exists $i^{\prime}>i$ such that $\left(p_{i i^{\prime \prime}} p_{i^{\prime \prime}}, p_{i}\right)<\mathcal{U}$ for $i^{\prime \prime}>i^{\prime}$.

An approximate resolution of a space $X$ is an approximate map $\boldsymbol{p}=\left\{p_{i}\right\}$ : $X \rightarrow \boldsymbol{X}$ of $X$ into an approximate sequence $\boldsymbol{X}=\left\{X_{i}, \mathcal{U}_{i}, p_{i i^{\prime}}\right\}$ which satisfies the following two conditions:

(R1) For each ANR $P, \mathcal{V} \in \operatorname{Cov}(P)$ and map $f: X \rightarrow P$, there exist $i \in \mathbb{N}$ and a map $g: X_{i} \rightarrow P$ such that $\left(g p_{i}, f\right)<\mathcal{V}$; and

(R2) For each ANR $P$ and $\mathcal{V} \in \operatorname{Cov}(P)$, there exists $\mathcal{V}^{\prime} \in \operatorname{Cov}(P)$ such that whenever $i \in \mathbb{N}$ and $g, g^{\prime}: X_{i} \rightarrow P$ are maps with $\left(g p_{i}, g^{\prime} p_{i}\right)<\mathcal{V}^{\prime}$, then $\left(g p_{i i^{\prime}}, g^{\prime} p_{i i^{\prime}}\right)<\mathcal{V}$ for some $i^{\prime}>i$.

If $\mathcal{C}$ is a collection of spaces, and if all $X_{i}$ belong to $\mathcal{C}$, then the approximate resolution $\boldsymbol{p}: X \rightarrow \boldsymbol{X}$ is called an approximate $\mathcal{C}$-resolution. Let POL denote the collection of polyhedra. Throughout the rest of the paper, an approximate resolution means an approximate POL-resolution unless otherwise stated.

It is known that an approximate map $\boldsymbol{p}=\left\{p_{i}\right\}: X \rightarrow \boldsymbol{X}=\left\{X_{i}, \mathcal{U}_{i}, p_{i i^{\prime}}\right\}$ is an approximate resolution of a space $X$ if and only if it satisfies the following two conditions:

(B1) For each $\mathcal{U} \in \operatorname{Cov}(X)$, there exists $i_{0} \in \mathbb{N}$ such that $p_{i}^{-1} \mathcal{U}_{i}<\mathcal{U}$ for $i>i_{0}$; and

(B2) For each $i \in \mathbb{N}$ and $\mathcal{U} \in \operatorname{Cov}\left(X_{i}\right)$, there exists $i_{0}>i$ such that $p_{i i^{\prime}}\left(X_{i^{\prime}}\right) \subseteq \operatorname{st}\left(p_{i}(X), \mathcal{U}\right)$ for $i^{\prime}>i_{0}$.

It is also known that every space $X$ admits an approximate resolution $\boldsymbol{p}=$ $\left\{p_{i}\right\}: X \rightarrow \boldsymbol{X}=\left\{X_{i}, \mathcal{U}_{i}, p_{i i^{\prime}}\right\}$ such that all $X_{i}$ are finite polyhedra ([14]), and that every connected space $X$ admits an approximate resolution $\boldsymbol{p}=\left\{p_{i}\right\}$ : $X \rightarrow \boldsymbol{X}=\left\{X_{i}, \mathcal{U}_{i}, p_{i i^{\prime}}\right\}$ such that all $X_{i}$ are connected finite polyhedra, and all $p_{i}$ and $p_{i i^{\prime}}$ are surjective ([4]).

Let $\boldsymbol{X}=\left\{X_{i}, \mathcal{U}_{i}, p_{i i^{\prime}}\right\}$ and $\boldsymbol{Y}=\left\{Y_{j}, \mathcal{V}_{j}, q_{j j^{\prime}}\right\}$ be approximate sequences of spaces. An approximate map $\boldsymbol{f}=\left\{f_{j}, f\right\}: \boldsymbol{X} \rightarrow \boldsymbol{Y}$ consists of an increasing function $f: \mathbb{N} \rightarrow \mathbb{N}$ and maps $f_{j}: X_{f(j)} \rightarrow Y_{j}, j \in \mathbb{N}$, with the following condition:

(AM) For any $j, j^{\prime} \in \mathbb{N}$ with $j<j^{\prime}$, there exists $i \in \mathbb{N}$ with $i>f\left(j^{\prime}\right)$ such that

$$
\left(q_{j j^{\prime}} f_{j^{\prime}} p_{f\left(j^{\prime}\right), i^{\prime}}, f_{j} p_{f(j), i^{\prime}}\right)<\mathrm{st} \mathcal{V}_{j} \text { for } i^{\prime}>i
$$


An approximate map $\boldsymbol{f}: \boldsymbol{X} \rightarrow \boldsymbol{Y}$ is said to be uniform if

$$
\mathcal{U}_{f(j)}<f^{-1} \mathcal{V}_{j} \text { for each } j \text {. }
$$

A map $f: X \rightarrow Y$ is a limit of $\boldsymbol{f}$ provided the following condition is satisfied:

(LAM) For each $j \in \mathbb{N}$ and $\mathcal{V} \in \operatorname{Cov}\left(Y_{j}\right)$, there exists $j^{\prime}>j$ such that

$$
\left(q_{j j^{\prime \prime}} f_{j^{\prime \prime}} p_{f\left(j^{\prime \prime}\right)}, q_{j} f\right)<\mathcal{V} \text { for } j^{\prime \prime}>j^{\prime} \text {. }
$$

For each map $f: X \rightarrow Y$, an approximate resolution of $f$ is a triple $(\boldsymbol{p}, \boldsymbol{q}, \boldsymbol{f})$ consisting of approximate resolutions $\boldsymbol{p}: X \rightarrow \boldsymbol{X}$ of $X$ and $\boldsymbol{q}: Y \rightarrow \boldsymbol{Y}$ of $Y$ and of an approximate map $\boldsymbol{f}: \boldsymbol{X} \rightarrow \boldsymbol{Y}$ with property (LAM). It is known that for any approximate resolutions $\boldsymbol{p}: X \rightarrow \boldsymbol{X}$ and $\boldsymbol{q}: Y \rightarrow \boldsymbol{Y}$, every map $f: X \rightarrow Y$ admits an approximate map $\boldsymbol{f}: \boldsymbol{X} \rightarrow \boldsymbol{Y}$ such that $(\boldsymbol{p}, \boldsymbol{q}, \boldsymbol{f})$ is an approximate resolution of $f$.

For each approximate sequence $\boldsymbol{X}=\left\{X_{i}, \mathcal{U}_{i}, p_{i i^{\prime}}\right\}$, let st $\boldsymbol{X}$ denote the approximate system $\left\{X_{i}\right.$, st $\left.\mathcal{U}_{i}, p_{i i^{\prime}}\right\}$. Then there is a natural approximate map $\boldsymbol{i}_{\boldsymbol{X}}=\left\{1_{X_{i}}\right\}: \boldsymbol{X} \rightarrow$ st $\boldsymbol{X}$, where $1_{X_{i}}: X_{i} \rightarrow X_{i}$ is the identity map. For each approximate map $\boldsymbol{p}=\left\{p_{i}\right\}: X \rightarrow \boldsymbol{X}=\left\{X_{i}, \mathcal{U}_{i}, p_{i i^{\prime}}\right\}$, the map st $\boldsymbol{p}=\left\{p_{i}\right\}: X \rightarrow$ st $\boldsymbol{X}=\left\{X_{i}\right.$, st $\left.\mathcal{U}_{i}, p_{i i^{\prime}}\right\}$ is an approximate map. Moreover, if $\boldsymbol{p}: X \rightarrow \boldsymbol{X}$ is an approximate resolution, so is st $\boldsymbol{p}: X \rightarrow$ st $\boldsymbol{X}$. For any approximate sequences $\boldsymbol{X}=\left\{X_{i}, \mathcal{U}_{i}, p_{i i^{\prime}}\right\}$ and $\boldsymbol{Y}=\left\{Y_{j}, \mathcal{V}_{j}, q_{j j^{\prime}}\right\}$ and for each approximate map $\boldsymbol{f}=\left\{f_{j}, f\right\}: \boldsymbol{X} \rightarrow \boldsymbol{Y}$, the map st $\boldsymbol{f}=\left\{f_{j}, f\right\}$ : st $\boldsymbol{X} \rightarrow$ st $\boldsymbol{Y}$ is an approximate map. Moreover, if $(\boldsymbol{f}, \boldsymbol{p}, \boldsymbol{q})$ is an approximate resolution of a map $f: X \rightarrow Y$, so is (st $\boldsymbol{f}$, st $\boldsymbol{p}$, st $\boldsymbol{q}$ ).

For each approximate map $\boldsymbol{f}=\left\{f_{j}, f\right\}: \boldsymbol{X} \rightarrow \boldsymbol{Y}$ where $\boldsymbol{p}=\left\{p_{i}\right\}: X \rightarrow$ $\boldsymbol{X}=\left\{X_{i}, \mathcal{U}_{i}, p_{i i^{\prime}}\right\}$ and $\boldsymbol{q}=\left\{q_{j}\right\}: Y \rightarrow \boldsymbol{Y}=\left\{Y_{j}, \mathcal{V}_{j}, q_{j j^{\prime}}\right\}$ are approximate resolutions, consider the following property:

$$
\begin{gathered}
(\mathrm{APS})(\forall j \in \mathbb{N})\left(\forall \mathcal{V} \in \operatorname{Cov}\left(Y_{j}\right)\right)\left(\exists j_{0}>j\right)\left(\forall j^{\prime}>j_{0}\right)\left(\exists j_{0}^{\prime}>j^{\prime}\right)\left(\forall j^{\prime \prime}>\right. \\
\left.j_{0}^{\prime}\right)\left(\exists i_{0}>f\left(j^{\prime}\right)\right)\left(\forall i>i_{0}\right): \\
q_{j j^{\prime \prime}}\left(Y_{j^{\prime \prime}}\right) \subseteq \operatorname{st}\left(q_{j j^{\prime}} f_{j^{\prime}} p_{f\left(j^{\prime}\right) i}\left(X_{i}\right), \mathcal{V}\right) .
\end{gathered}
$$

Then we have

TheOREm 2.1. Let $f: X \rightarrow Y$ be a map, and $\boldsymbol{f}=\left\{f_{j}\right\}: \boldsymbol{X} \rightarrow \boldsymbol{Y}$ be an approximate map such that $(\boldsymbol{f}, \boldsymbol{p}, \boldsymbol{q})$ is an approximate resolution of $f$, where $\boldsymbol{p}=\left\{p_{i}\right\}: X \rightarrow \boldsymbol{X}=\left\{X_{i}, \mathcal{U}_{i}, p_{i i^{\prime}}\right\}$ and $\boldsymbol{q}=\left\{q_{j}\right\}: Y \rightarrow \boldsymbol{Y}=\left\{Y_{j}, \mathcal{V}_{j}, q_{j j^{\prime}}\right\}$ are approximate resolutions of $X$ and $Y$, respectively. Then $f$ is surjective if and only if $\boldsymbol{f}$ satisfies (APS).

Proof. See [10]

Note that if $\boldsymbol{f}: \boldsymbol{X} \rightarrow \boldsymbol{Y}$ has property (APS), so does st $\boldsymbol{f}:$ st $\boldsymbol{X} \rightarrow$ st $\boldsymbol{Y}$.

Following the approach of Alexandroff and Urysohn (see [1] and [11, 2$16]$ ), given a space $X$ and a normal sequence $\mathbb{U}$ on $X$, we define a metric $\mathrm{d}_{\mathbb{U}}$ on $X$. 
A family $\mathbb{U}=\left\{\mathcal{U}_{i}: i \in \mathbb{N}\right\}$ of open coverings on a space $X$ is said to be a normal sequence provided st $\mathcal{U}_{i+1}<\mathcal{U}_{i}$ for each $i$. Let $\Sigma \mathbb{U}$ denote the normal sequence $\left\{\mathcal{V}_{i}: \mathcal{V}_{i}=\mathcal{U}_{i+1}, i \in \mathbb{N}\right\}$ and st $\mathbb{U}$ the normal sequence $\left\{\right.$ st $\left.\mathcal{U}_{i}: i \in \mathbb{N}\right\}$. For any normal sequences $\mathbb{U}=\left\{\mathcal{U}_{i}\right\}$ and $\mathbb{V}=\left\{\mathcal{V}_{i}\right\}$, we write $\mathbb{U}<\mathbb{V}$ provided $\mathcal{U}_{i}<\mathcal{V}_{i}$ for each $i$. Let $\Sigma^{0} \mathbb{U}=\mathbb{U}$, and for each $n \in \mathbb{N}$, let $\Sigma^{n} \mathbb{U}=\Sigma\left(\Sigma^{n-1} \mathbb{U}\right)$, and also let $\mathrm{st}^{0} \mathbb{U}=\mathbb{U}$ and $\left.\mathrm{st}^{n} \mathbb{U}=\mathrm{st}_{(\mathrm{st}}{ }^{n-1} \mathbb{U}\right)$. For each map $f: X \rightarrow Y$ and for each normal sequence $\mathbb{V}=\left\{V_{i}\right\}$, let $f^{-1} \mathbb{V}=\left\{f^{-1} \mathcal{V}_{i}\right\}$. For each closed subset $A$ of $X$ and for each normal sequence $\mathbb{U}=\left\{\mathcal{U}_{i}\right\}$ on $X$, let $\mathbb{U} \mid A=\left\{\mathcal{U}_{i} \mid A\right\}$.

Given a normal sequence $\mathbb{U}=\left\{\mathcal{U}_{i}\right\}$ on $X$, we define a function $\mathcal{D}_{\mathbb{U}}$ : $X \times X \rightarrow \mathbb{R}_{\geq 0}$ by

$$
\mathcal{D}_{\mathbb{U}}\left(x, x^{\prime}\right)=\left\{\begin{array}{cl}
9, & \text { if }\left(x, x^{\prime}\right) \nless \mathcal{U}_{1} ; \\
\frac{1}{3^{i-2},}, & \text { if }\left(x, x^{\prime}\right)<\mathcal{U}_{i} \text { but }\left(x, x^{\prime}\right) \nless \mathcal{U}_{i+1} ; \\
0, & \text { if }\left(x, x^{\prime}\right)<\mathcal{U}_{i} \text { for all } i \in \mathbb{N},
\end{array}\right.
$$

and a function $\mathrm{d}_{\mathbb{U}}: X \times X \rightarrow \mathbb{R}_{\geq 0}$ by

$$
\mathrm{d}_{\mathbb{U}}\left(x, x^{\prime}\right)=\inf \left\{\mathcal{D}_{\mathbb{U}}\left(x, x_{1}\right)+\mathcal{D}_{\mathbb{U}}\left(x_{1}, x_{2}\right)+\cdots+\mathcal{D}_{\mathbb{U}}\left(x_{n}, x^{\prime}\right)\right\}
$$

where the infimum is taken over all points $x_{1}, x_{2}, \ldots, x_{n}$ in $X$ and $\mathbb{R}_{\geq 0}$ denotes the set of nonnegative real numbers. Then the function $\mathrm{d}_{\mathbb{U}}: X \times X \rightarrow \mathbb{R}_{\geq 0}$ defines a pseudometric on $X$ with the property that

$$
\operatorname{st}\left(x, \mathcal{U}_{i+3}\right) \subseteq \mathrm{U}_{\mathrm{d}_{\mathbb{U}}}\left(x, \frac{1}{3^{i}}\right) \subseteq \operatorname{st}\left(x, \mathcal{U}_{i}\right) \text { for each } x \in X \text { and } i .
$$

Moreover, if $\mathbb{U}$ has the following property:

(B) $\left\{\operatorname{st}\left(x, \mathcal{U}_{i}\right): i \in \mathbb{N}\right\}$ is a base at $x$ for each $x \in X$,

then $\mathrm{d}_{\mathbb{U}}$ defines a metric on $X$, which we call the metric induced by the normal sequence $\mathbb{U}$. In particular, if $\mathbb{U}=\left\{\mathcal{U}_{i}\right\}$ is the normal sequence such that $\mathcal{U}_{i}=\left\{\mathrm{U}_{\mathrm{d}}\left(x, \frac{1}{3^{i}}\right): x \in X\right\}$, then the metric $\mathrm{d}_{\mathbb{U}}$ induced by the normal sequence $\mathbb{U}$ induces the uniformity which is isomorphic to that induced by the metric $\mathrm{d}$.

Proposition 2.2. Let $X$ be a space, and let $\mathbb{U}=\left\{\mathcal{U}_{i}\right\}$ and $\mathbb{V}=\left\{\mathcal{V}_{i}\right\}$ be normal sequences on $X$. Then we have the following properties:

1) If $A$ is a closed subset of $X$, then $\mathrm{d}_{\mathbb{U} \mid A}\left(x, x^{\prime}\right) \geq \mathrm{d}_{\mathbb{U}}\left(x, x^{\prime}\right)$ for all $x, x^{\prime} \in A$.

2) If $\mathbb{U}<\mathbb{V}$, then $\mathrm{d}_{\mathbb{U}}\left(x, x^{\prime}\right) \geq \mathrm{d}_{\mathbb{V}}\left(x, x^{\prime}\right)$ for all $x, x^{\prime} \in X$.

3) $\mathrm{d}_{\Sigma \mathbb{U}}\left(x, x^{\prime}\right)=3 \mathrm{~d}_{\mathbb{U}}\left(x, x^{\prime}\right)$ for all $x, x^{\prime} \in X$.

4) $\mathrm{d}_{\mathrm{st} \mathbb{U}}\left(x, x^{\prime}\right) \leq \mathrm{d}_{\mathbb{U}}\left(x, x^{\prime}\right) \leq 3 \mathrm{~d}_{\mathrm{st} \mathbb{U}}\left(x, x^{\prime}\right)$ for all $x, x^{\prime} \in X$.

For each approximate resolution $\boldsymbol{p}=\left\{p_{i}\right\}: X \rightarrow \boldsymbol{X}=\left\{X_{i}, \mathcal{U}_{i}, p_{i i^{\prime}}\right\}$, consider the following three properties:

(U) $\mathrm{st}^{2} \mathcal{U}_{j}<p_{i j}^{-1} \mathcal{U}_{i}$ for $i<j$;

(A) $\left(p_{i j} p_{j}, p_{i}\right)<\mathcal{U}_{i}$ for $i<j$; and

(NR) $p_{j}^{-1}$ st $\mathcal{U}_{j}<p_{i}^{-1} \mathcal{U}_{i}$ for $i<j$. 
An approximate resolution $\boldsymbol{p}=\left\{p_{i}\right\}: X \rightarrow \boldsymbol{X}=\left\{X_{i}, \mathcal{U}_{i}, p_{i i^{\prime}}\right\}$ is said to be admissible provided it possesses properties (U), (A), (NR) and the family $\mathbb{U}=\left\{p_{i}^{-1} \mathcal{U}_{i}\right\}$ has property (B).

Proposition 2.3. Let $\boldsymbol{p}=\left\{p_{i}\right\}: X \rightarrow \boldsymbol{X}=\left\{X_{i}, \mathcal{U}_{i}, p_{i i^{\prime}}\right\}$ be an admissible approximate resolution of $X$. Then the following properties hold:

1) The family $\mathbb{U}_{k}=\left\{p_{i}^{-1} \mathrm{st}^{k} \mathcal{U}_{i}: i \in \mathbb{N}\right\}$ forms a normal sequence on $X$ for $k \geq 0$;

2) The approximate resolution $\mathrm{st}^{k} \boldsymbol{p}=\left\{p_{i}\right\}: X \rightarrow \mathrm{st}^{k} \boldsymbol{X}=\left\{X_{i}, \mathrm{st}^{k} \mathcal{U}_{i}, p_{i i^{\prime}}\right\}$ is admissible for $k \geq 1$.

For any approximate resolution $\boldsymbol{p}=\left\{p_{i}\right\}: X \rightarrow \boldsymbol{X}=\left\{X_{i}, \mathcal{U}_{i}, p_{i i^{\prime}}\right\}$, we can always find an admissible approximate resolution $\boldsymbol{p}^{\prime}=\left\{p_{k_{i}}\right\}: X \rightarrow \boldsymbol{X}^{\prime}=$ $\left\{X_{k_{i}}, \mathcal{U}_{k_{i}}, p_{k_{i} k_{j}}\right\}$ by taking a subsystem.

Let $\boldsymbol{p}: X \rightarrow \boldsymbol{X}=\left\{X_{i}, \mathcal{U}_{i}, p_{i i^{\prime}}\right\}$ be any admissible approximate resolution of a space $X$. Then for any $x, x^{\prime} \in X$, we define a function $\mathcal{D}_{p}: X \times X \rightarrow \mathbb{R}_{\geq 0}$ by

$$
\mathcal{D}_{\boldsymbol{p}}\left(x, x^{\prime}\right)=\left\{\begin{array}{cl}
9, & \text { if }\left(p_{i}(x), p_{i}\left(x^{\prime}\right)\right) \nless \mathcal{U}_{i} \text { for any } i ; \\
\frac{1}{3^{i-2},}, & \text { if }\left(p_{i}(x), p_{i}\left(x^{\prime}\right)\right)<\mathcal{U}_{i} \text { but }\left(p_{i}(x), p_{i}\left(x^{\prime}\right)\right) \nless \mathcal{U}_{i+1} ; \\
0, & \text { if }\left(p_{i}(x), p_{i}\left(x^{\prime}\right)\right)<\mathcal{U}_{i} \text { for all } i,
\end{array}\right.
$$

and a function $\mathrm{d}_{\boldsymbol{p}}: X \times X \rightarrow \mathbb{R}_{\geq 0}$ by

$$
\mathrm{d}_{\boldsymbol{p}}\left(x, x^{\prime}\right)=\inf \left\{\mathcal{D}_{\boldsymbol{p}}\left(x, x_{1}\right)+\mathcal{D}_{\boldsymbol{p}}\left(x_{1}, x_{2}\right)+\cdots+\mathcal{D}_{\boldsymbol{p}}\left(x_{n}, x^{\prime}\right)\right\}
$$

where the infimum is taken over all finite collections of points $x_{1}, x_{2}, \ldots, x_{n}$ of $X$. Note that $\mathrm{d}_{\boldsymbol{p}}\left(x, x^{\prime}\right)=\mathrm{d}_{\mathbb{U}}\left(x, x^{\prime}\right)$ for any $x, x^{\prime} \in X$, where $\mathbb{U}=\left\{p_{i}^{-1} \mathcal{U}_{i}\right\}$.

For each approximate resolution $\boldsymbol{p}=\left\{p_{i}\right\}: X \rightarrow \boldsymbol{X}=\left\{X_{i}, \mathcal{U}_{i}, p_{i i^{\prime}}\right\}$, we define the approximate sequence $\Sigma \boldsymbol{X}$ as $\left\{Z_{i}, \mathcal{W}_{i}, r_{i i^{\prime}}\right\}$ where $Z_{i}=X_{i+1}$, $\mathcal{W}_{i}=\mathcal{U}_{i+1}, r_{i i^{\prime}}=p_{i+1, i^{\prime}+1}: Z_{i^{\prime}} \rightarrow Z_{i}$ and the approximate resolution $\Sigma \boldsymbol{p}$ as $\left\{r_{i}: i \in \mathbb{N}\right\}: X \rightarrow \Sigma \boldsymbol{X}$ where $r_{i}=p_{i+1}: X \rightarrow X_{i+1}$. Let $\Sigma^{0} \boldsymbol{X}=\boldsymbol{X}$ and $\Sigma^{0} \boldsymbol{p}=\boldsymbol{p}$, and for each $i \in \mathbb{N}$, let $\Sigma^{n} \boldsymbol{X}=\Sigma\left(\Sigma^{n-1} \boldsymbol{X}\right)$ and $\Sigma^{n} \boldsymbol{p}=\Sigma\left(\Sigma^{n-1} \boldsymbol{p}\right)$.

Proposition 2.4. Let $X$ be a space, and let $\boldsymbol{p}=\left\{p_{i}\right\}: X \rightarrow \boldsymbol{X}=$ $\left\{X_{i}, \mathcal{U}_{i}, p_{i i^{\prime}}\right\}$ be an admissible approximate resolution of $X$. Then

1) $\mathrm{d}_{\Sigma^{n} \boldsymbol{p}}\left(x, x^{\prime}\right)=3^{n} \mathrm{~d}_{\boldsymbol{p}}\left(x, x^{\prime}\right)$ for $x, x^{\prime} \in X$ and for each $n \in \mathbb{N}$; and

2) $\mathrm{d}_{\text {st } \boldsymbol{p}}\left(x, x^{\prime}\right) \leq \mathrm{d}_{\boldsymbol{p}}\left(x, x^{\prime}\right) \leq 3 \mathrm{~d}_{\text {st } \boldsymbol{p}}\left(x, x^{\prime}\right)$ for $x, x^{\prime} \in X$.

Throughout the paper, approximate resolutions are assumed to be admissible unless otherwise stated.

\section{BI-LIPSCHITZ MAPS}

In this section we consider bi-Lipschitz maps with respect to the metrics induced by approximate resolutions. In particular, we give a characterization in terms of approximate resolutions. But first, we consider normal sequences. 
Let $X$ and $Y$ be spaces with normal sequences $\mathbb{U}=\left\{U_{i}\right\}$ and $\mathbb{V}=\left\{V_{i}\right\}$, respectively. Then a map $f: X \rightarrow Y$ is called a $(\mathbb{U}, \mathbb{V})$-Lipschitz map provided there exists a constant $\alpha>0$ such that

$$
\mathrm{d}_{\mathbb{V}}\left(f(x), f\left(x^{\prime}\right)\right) \leq \alpha \mathrm{d}_{\mathbb{U}}\left(x, x^{\prime}\right) \text { for } x, x^{\prime} \in X,
$$

and a $(\mathbb{U}, \mathbb{V})$-bi-Lipschitz map provided there exist constants $\alpha_{1}, \alpha_{2}>0$ such that

$$
\alpha_{1} \mathrm{~d}_{\mathbb{U}}\left(x, x^{\prime}\right) \leq \mathrm{d}_{\mathbb{V}}\left(f(x), f\left(x^{\prime}\right)\right) \leq \alpha_{2} \mathrm{~d}_{\mathbb{U}}\left(x, x^{\prime}\right) \text { for } x, x^{\prime} \in X .
$$

TheOrem 3.1. Let $X$ and $Y$ be spaces with normal sequences $\mathbb{U}=\left\{U_{i}\right\}$ and $\mathbb{V}=\left\{V_{i}\right\}$, respectively, and let $f: X \rightarrow Y$ be a map. Consider the following properties:

1) $\mathrm{d}_{\mathbb{U}}\left(x, x^{\prime}\right) \leq \mathrm{d}_{\mathbb{V} \mid f(X)}\left(f(x), f\left(x^{\prime}\right)\right)$ for $x, x^{\prime} \in X$;

2) $f^{-1} \mathbb{V}<\mathbb{U}$; and

3) $f^{-1} \Sigma^{4} \mathbb{V}<\mathbb{U}$.

Then the implications 2) $\Rightarrow$ 1) $\Rightarrow$ 3) hold.

Proof. To see 2) $\Rightarrow 1)$, let $x, x^{\prime} \in X$, and let $y_{0}=f(x), y_{1}, y_{2}, \ldots, y_{n}=$ $f\left(x^{\prime}\right)$ be any points in $f(X)$. Say $y_{i}=f\left(x_{i}\right)$ for some $x_{i} \in X$. If $\mathcal{D}_{\mathbb{V} \mid f(X)}\left(f\left(x_{i}\right), f\left(x_{i+1}\right)\right)=\frac{1}{3^{k_{i}-2}}$ for some $\left.k_{i} \geq 0,2\right)$ implies that $\mathcal{D}_{\mathbb{U}}\left(x_{i}, x_{i+1}\right) \leq \frac{1}{3^{k_{i}-2}}$. Hence 1) holds. To see 1) $\left.\Rightarrow 3\right)$, let $i \in \mathbb{N}$, and let $V \in$ $\mathcal{V}_{i+4}$. Take $x \in f^{-1}(V)$. Then property (2.1) implies $V \subseteq U_{\mathrm{dV}}\left(f(x), \frac{1}{3^{i+1}}\right)$. If $x^{\prime} \in f^{-1}(V)$, then $f\left(x^{\prime}\right) \in V \subseteq U_{\mathrm{dV}_{\mathrm{V}}}\left(f(x), \frac{1}{3^{i+1}}\right)$. So, 1) and property (2.1) imply $x^{\prime} \in U_{\mathrm{d}_{\mathbb{U}}}\left(x, \frac{1}{3^{i+1}}\right) \subseteq \operatorname{st}\left(x, \mathcal{U}_{i+1}\right) \subseteq U$ for some $U \in \mathcal{U}_{i}$, showing $f^{-1}(V) \subseteq U$.

THEOREM 3.2. Under the same setting as in Theorem 3.1, consider the following property for $m \in \mathbb{Z}$ :

$(\mathrm{L})^{m} \mathrm{~d}_{\mathbb{U}}\left(x, x^{\prime}\right) \leq 3^{m} \mathrm{~d}_{\mathbb{V} \mid f(X)}\left(f(x), f\left(x^{\prime}\right)\right)$ for $x, x^{\prime} \in X ;$

and for $m, n \geq 0$, the following two properties:

$(\mathrm{M})^{m, n} f^{-1} \mathrm{st}^{m} \mathbb{V}<\Sigma^{n} \mathbb{U} ;$ and

$(\mathrm{N})^{m, n} f^{-1} \Sigma^{m} \mathbb{V}<\Sigma^{n} \mathbb{U}$.

Then we have the following implications for $m, n \geq 0$ :

1) $(\mathrm{L})^{m} \Rightarrow(\mathrm{N})^{m+4,0}$;

2) $(\mathrm{L})^{-m} \Rightarrow(\mathrm{N})^{4, m}$

3) $(\mathrm{N})^{m, n} \Rightarrow(\mathrm{L})^{m-n}$;

4) $(\mathrm{M})^{m, n} \Rightarrow(\mathrm{L})^{m-n}$.

Proof. To see 1), note that $(\mathrm{L})^{m}$ means

$$
\mathrm{d}_{\mathbb{U}}\left(x, x^{\prime}\right) \leq \mathrm{d}_{\Sigma^{m} \mathbb{V} \mid f(X)}\left(f(x), f\left(x^{\prime}\right)\right) \text { for } x, x^{\prime} \in X .
$$

But this together with Theorem 3.1 implies $f^{-1} \Sigma^{4}\left(\Sigma^{m} \mathbb{V}\right)<\mathbb{U}$, which means $\left.(\mathrm{N})^{m+4,0} .2\right)$ is proven similarly to 1$)$. To show 3$)$, note that $(\mathrm{N})^{m, n}$ together 
with Proposition 2.2 3) and Theorem 3.1 implies

$$
\begin{aligned}
3^{n} \mathrm{~d}_{\mathbb{U}}\left(x, x^{\prime}\right)=\mathrm{d}_{\Sigma^{n} \mathbb{U}}\left(x, x^{\prime}\right) & \leq \mathrm{d}_{\Sigma^{m} \mathbb{V} \mid f(X)}\left(f(x), f\left(x^{\prime}\right)\right) \\
& =3^{m} \mathrm{~d}_{\mathbb{V} \mid f(X)}\left(f(x), f\left(x^{\prime}\right)\right) \text { for } x, x^{\prime} \in X,
\end{aligned}
$$

which means $(\mathrm{L})^{m-n}$. To show 4$)$, note that $(\mathrm{M})^{m, n}$ together with Proposition 2.23$), 4$ ) implies

$$
\begin{aligned}
3^{n} \mathrm{~d}_{\mathbb{U}}\left(x, x^{\prime}\right)=\mathrm{d}_{\Sigma^{n} \mathbb{U}}\left(x, x^{\prime}\right) & \leq \mathrm{d}_{\mathrm{st}^{m} \mathbb{V} \mid f(X)}\left(f(x), f\left(x^{\prime}\right)\right) \\
& \leq 3^{m} \mathrm{~d}_{\mathbb{V} \mid f(X)}\left(f(x), f\left(x^{\prime}\right)\right) \text { for } x, x^{\prime} \in X,
\end{aligned}
$$

which means $(\mathrm{L})^{m-n}$.

Let $f: X \rightarrow Y$ be a map and let $\boldsymbol{f}: \boldsymbol{X} \rightarrow \boldsymbol{Y}$ be an approximate map such that $(\boldsymbol{f}, \boldsymbol{p}, \boldsymbol{q})$ is an approximate resolution of $f$, where $\boldsymbol{p}: X \rightarrow \boldsymbol{X}$ and $\boldsymbol{q}: Y \rightarrow \boldsymbol{Y}$ are approximate resolutions of $X$ and $Y$, respectively. Then a map $f: X \rightarrow Y$ is called a $(\boldsymbol{p}, \boldsymbol{q})$-Lipschitz map provided there exists a constant $\alpha>0$ such that

$$
\mathrm{d}_{\boldsymbol{q}}\left(f(x), f\left(x^{\prime}\right)\right) \leq \alpha \mathrm{d}_{\boldsymbol{p}}\left(x, x^{\prime}\right) \text { for } x, x^{\prime} \in X,
$$

and a $(\boldsymbol{p}, \boldsymbol{q})$-biLipschitz map provided there exist constants $\alpha_{1}, \alpha_{2}>0$ such that

$$
\alpha_{1} \mathrm{~d}_{\boldsymbol{p}}\left(x, x^{\prime}\right) \leq \mathrm{d}_{\boldsymbol{q}}\left(f(x), f\left(x^{\prime}\right)\right) \leq \alpha_{2} \mathrm{~d}_{\boldsymbol{p}}\left(x, x^{\prime}\right) \text { for } x, x^{\prime} \in X .
$$

TheOREm 3.3. Let $X$ and $Y$ be spaces, and let $f: X \rightarrow Y$ be a surjective map. Also let $\boldsymbol{p}=\left\{p_{i}\right\}: X \rightarrow \boldsymbol{X}=\left\{X_{i}, \mathcal{U}_{i}, p_{i i^{\prime}}\right\}$ and $\boldsymbol{q}=\left\{q_{j}\right\}: Y \rightarrow$ $\boldsymbol{Y}=\left\{Y_{j}, \mathcal{V}_{j}, q_{j j^{\prime}}\right\}$ be approximate resolutions of $X$ and $Y$, respectively, and let $\boldsymbol{f}=\left\{f_{j}\right\}: \boldsymbol{X} \rightarrow \boldsymbol{Y}$ be an approximate map such that $(\boldsymbol{f}, \boldsymbol{p}, \boldsymbol{q})$ is an approximate resolution of $f$. Consider the following property for $m \in \mathbb{Z}$ :

$(\operatorname{Lip})^{m} 3^{m} \mathrm{~d}_{\boldsymbol{p}}\left(x, x^{\prime}\right) \leq \mathrm{d}_{\boldsymbol{q}}\left(f(x), f\left(x^{\prime}\right)\right)$ for $x, x^{\prime} \in X ;$

and the following two properties for $m \geq 0$,

$(\mathrm{ALip})^{m}$ For each $i$, there exists $j_{0}>i$ with the property that each $j>j_{0}$ admits $i_{0}>i+m, f(j)$ such that

$$
p_{f(j), i^{\prime}}^{-1} f_{j}^{-1} q_{i j}^{-1} \mathcal{V}_{i}<p_{i+m, i^{\prime}}^{-1} \mathcal{U}_{i+m} \text { for } i^{\prime}>i_{0} ; \text { and }
$$

(ALip) ${ }^{-m}$ For each $i$, there exists $j_{0}>i+m$ with the property that each $j>j_{0}$ admits $i_{0}>i, f(j)$ such that

$$
p_{f(j), i^{\prime}}^{-1} f_{j}^{-1} q_{i+m, j}^{-1} \mathcal{V}_{i+m}<p_{i i^{\prime}}^{-1} \mathcal{U}_{i} \text { for } i^{\prime}>i_{0} .
$$

Then the following implications hold for each $m \in \mathbb{Z}$ :

1) $(\mathrm{ALip})^{m}$ for st $\boldsymbol{f}:$ st $\boldsymbol{X} \rightarrow$ st $\boldsymbol{Y} \Rightarrow(\mathrm{Lip})^{m-2}$ for $\boldsymbol{p}$ and $\boldsymbol{q}$;

2) If each $p_{i}$ is surjective, (Lip) ${ }^{m+2}$ for $\boldsymbol{p}$ and $\boldsymbol{q} \Rightarrow(\mathrm{ALip})^{m}$ for st $\boldsymbol{f}$ : st $\boldsymbol{X} \rightarrow$ st $\boldsymbol{Y}$. 
Proof. We can assume $m \geq 0$ since the argument for $m<0$ is similar. Suppose (ALip) ${ }^{m}$ holds for st $\boldsymbol{f}:$ st $\boldsymbol{X} \rightarrow$ st $\boldsymbol{Y}$, and let $i \in \mathbb{N}$. Take $\mathcal{V} \in$ $\operatorname{Cov}\left(Y_{i}\right)$ such that st $\mathcal{V}<\mathcal{V}_{i}$, and take $j_{0}>i$ as in (ALip) ${ }^{m}$. By (LAM), there exists $j_{1}>j_{0}$ such that

$$
\left(q_{i} f, q_{i j} f_{j} p_{f(j)}\right)<\mathcal{V} \text { for } j>j_{1} .
$$

Fix $j>j_{1}$, and for this $j$, take $i_{0}>i+m, f(j)$ as in (ALip) ${ }^{m}$. By (AS), there exists $i^{\prime}>i_{0}$ such that

$$
\left(p_{f(j)}, p_{f(j), i^{\prime}} p_{i^{\prime}}\right)<f_{j}^{-1} q_{i j}^{-1} \mathcal{V}
$$

and

$$
\left(p_{i+m}, p_{i+m, i^{\prime}} p_{i^{\prime}}\right)<\mathcal{U}_{i+m} .
$$

Then, for each $V \in \mathcal{V}_{i}$, by (3.1), (3.2), (ALip) ${ }^{m}$ and (3.3), for some $U \in \mathcal{U}_{i+m}$,

$$
\begin{aligned}
f^{-1} q_{i}^{-1}(V) & \subseteq p_{f(j)}^{-1} f_{j}^{-1} q_{i j}^{-1}(\operatorname{st}(V, \mathcal{V})) \\
& \subseteq p_{i^{\prime}}^{-1} p_{f(j), i^{\prime}}^{-1} f_{j}^{-1} q_{i j}^{-1}(\operatorname{st}(\operatorname{st}(V, \mathcal{V}), \mathcal{V})) \\
& \subseteq p_{i^{\prime}}^{-1} p_{f(j), i^{\prime}}^{-1} f_{j}^{-1} q_{i j}^{-1}\left(\operatorname{st}\left(V, \mathcal{V}_{i}\right)\right) \\
& \subseteq p_{i^{\prime}}^{-1} p_{i+m, i^{\prime}}^{-1}\left(\operatorname{st}\left(U, \mathcal{U}_{i+m}\right)\right) \\
& \subseteq p_{i+m}^{-1}\left(\operatorname{st}\left(\operatorname{st}\left(U, \mathcal{U}_{i+m}\right), \mathcal{U}_{i+m}\right) .\right.
\end{aligned}
$$

This means $f^{-1} q_{i}^{-1} \mathcal{V}_{i}<p_{i+m}^{-1} \mathrm{st}^{2} \mathcal{U}_{i+m}$, and hence $f^{-1} \mathbb{V}<\Sigma^{m} \mathbb{U}$ where $\mathbb{U}=$ $\left\{p_{i}^{-1} \mathrm{st}^{2} \mathcal{U}_{i}\right\}$ and $\mathbb{V}=\left\{q_{i}^{-1} \mathcal{V}_{i}\right\}$. By Theorem 3.2,

$$
\mathrm{d}_{\Sigma^{m} \mathbb{U}}\left(x, x^{\prime}\right) \leq \mathrm{d}_{\mathbb{V}}\left(f(x), f\left(x^{\prime}\right)\right) \text { for } x, x^{\prime} \in X,
$$

which means $(\operatorname{Lip})^{m}$ for $\mathrm{st}^{2} \boldsymbol{p}$ and $\boldsymbol{q}$. This together with Proposition 2.4 implies (Lip) ${ }^{m-2}$ for $\boldsymbol{p}$ and $\boldsymbol{q}$, verifying 1 ).

To see 2), first note that (Lip) ${ }^{m+2}$ for $\boldsymbol{p}$ and $\boldsymbol{q}$ means (Lip) ${ }^{m}$ for $\boldsymbol{p}$ and $\mathrm{st}^{2} \boldsymbol{q}$. Suppose now that all $p_{i}$ are surjective, and suppose (Lip) ${ }^{m}$ for $\boldsymbol{p}$ and $\mathrm{st}^{2} \boldsymbol{q}$. Let $i \in \mathbb{N}$, and take $\mathcal{V} \in \operatorname{Cov}\left(Y_{i}\right)$ such that st $\mathcal{V}<\mathcal{V}_{i}$. Then by (LAM) there exists $j_{0}>i$ such that

$$
\left(q_{i} f, q_{i j} f_{j} p_{f(j)}\right)<\mathcal{V} \text { for each } j>j_{0} .
$$

Fix $j>j_{0}$. Then by (AS) there exists $i_{0}>i+m, f(j)$ such that for each $i^{\prime}>i_{0}$

$$
\left(p_{i+m}, p_{i+m, i^{\prime}} p_{i^{\prime}}\right)<\mathcal{U}_{i+m},
$$

and

$$
\left(p_{f(j)}, p_{f(j), i^{\prime}} p_{i^{\prime}}\right)<f_{j}^{-1} q_{i j}^{-1} \mathcal{V}
$$


Then, for each $i^{\prime}>i_{0}$ and for each $V \in \mathcal{V}_{i}$, by (3.6), (3.4), (Lip) ${ }^{m}$ for $\boldsymbol{p}$ and $\mathrm{st}^{2} \boldsymbol{q}$, and (3.5), for some $U \in \mathcal{U}_{i+m}$,

$$
\begin{aligned}
p_{i^{\prime}}^{-1} p_{f(j), i^{\prime}}^{-1} f_{j}^{-1} q_{i j}^{-1}\left(\operatorname{st}\left(V, \mathcal{V}_{i}\right)\right) & \subseteq p_{f(j)}^{-1} f_{j}^{-1} q_{i j}^{-1}\left(\operatorname{st}\left(\operatorname{st}\left(V, \mathcal{V}_{i}\right), \mathcal{V}\right)\right. \\
& \subseteq f^{-1} q_{i}^{-1}\left(\operatorname{st}\left(\operatorname{st}\left(\operatorname{st}\left(V, \mathcal{V}_{i}\right), \mathcal{V}\right), \mathcal{V}\right)\right) \\
& \subseteq f^{-1} q_{i}^{-1}\left(\operatorname{st}\left(\operatorname{st}\left(V, \mathcal{V}_{i}\right), \mathcal{V}_{i}\right)\right) \\
& \subseteq p_{i+m}^{-1}(U) \\
& \subseteq p_{i^{\prime}}^{-1} p_{i+m, i^{\prime}}^{-1}\left(\operatorname{st}\left(U, \mathcal{U}_{i+m}\right)\right)
\end{aligned}
$$

Since each $p_{i^{\prime}}$ is surjective,

$$
p_{f(j) i^{\prime}}^{-1} f_{j}^{-1} q_{i j}^{-1}\left(\operatorname{st}\left(V, \mathcal{V}_{i}\right)\right) \subseteq p_{i+m, i^{\prime}}^{-1}\left(\operatorname{st}\left(U, \mathcal{U}_{i+m}\right)\right),
$$

proving (ALip) ${ }^{m}$ for st $\boldsymbol{f}:$ st $\boldsymbol{X} \rightarrow$ st $\boldsymbol{Y}$. This verifies 2).

Recall the following two results concerning Lipschitz maps from $[8,9]$ :

Theorem 3.4. Let $X$ and $Y$ be spaces with normal sequences $\mathbb{U}=\left\{\mathcal{U}_{i}\right\}$ and $\mathbb{V}=\left\{\mathcal{V}_{i}\right\}$, respectively, and let $f: X \rightarrow Y$ be a map. Consider the following statements:

$(\mathrm{L})_{m} \mathrm{~d}_{\mathbb{V}}\left(f(x), f\left(x^{\prime}\right)\right) \leq 3^{m} \mathrm{~d}_{\mathbb{U}}\left(x, x^{\prime}\right)$ for $x, x^{\prime} \in X$;

$(\mathrm{M})_{m, n} \Sigma^{m} \mathbb{U}<f^{-1} \mathrm{st}^{n} \mathbb{V}$; and

$(\mathrm{N})_{m, n} \Sigma^{m} \mathbb{U}<f^{-1} \Sigma^{n} \mathbb{V}$.

Then the following implications hold for any $m, n \geq 0$ :

1) $(\mathrm{M})_{m, n} \Rightarrow(\mathrm{L})_{m+n}$;

2) $(\mathrm{N})_{m, n} \Rightarrow(\mathrm{L})_{n-m}$;

3) $(\mathrm{L})_{m} \Rightarrow(\mathrm{M})_{m+4,0}=(\mathrm{N})_{m+4,0}$; and

4) $(\mathrm{L})_{-m} \Rightarrow(\mathrm{N})_{4, m}$.

and

Theorem 3.5. Let $X$ and $Y$ be spaces, and let $f: X \rightarrow Y$ be a map. Also let $\boldsymbol{p}=\left\{p_{i}\right\}: X \rightarrow \boldsymbol{X}=\left\{X_{i}, \mathcal{U}_{i}, p_{i i^{\prime}}\right\}$ and $\boldsymbol{q}=\left\{q_{j}\right\}: Y \rightarrow \boldsymbol{Y}=\left\{Y_{j}, \mathcal{V}_{j}, q_{j j^{\prime}}\right\}$ be approximate resolutions of $X$ and $Y$, respectively, and let $\boldsymbol{f}=\left\{f_{j}\right\}: \boldsymbol{X} \rightarrow$ $\boldsymbol{Y}$ be an approximate map such that $(\boldsymbol{f}, \boldsymbol{p}, \boldsymbol{q})$ is an approximate resolution of $f$. For each $m \in \mathbb{Z}$, consider the following property:

$(\text { Lip })_{m} \mathrm{~d}_{\boldsymbol{q}}\left(f(x), f\left(x^{\prime}\right)\right) \leq 3^{m} \mathrm{~d}_{\boldsymbol{p}}\left(x, x^{\prime}\right)$ for $x, x^{\prime} \in X$, and for $m \geq 0$, consider the following two properties:

(ALip) $)_{m}$ For each $i$, there exists $j_{0}>i$ with the property that each $j>j_{0}$ admits $i_{0}>f(j), i+m$ such that, for each $i^{\prime}>i_{0}$,

$$
p_{i+m, i^{\prime}}^{-1} \mathcal{U}_{i+m}<p_{f(j), i^{\prime}}^{-1} f_{j}^{-1} q_{i j}^{-1} \mathcal{V}_{i},
$$

and 
(ALip) $-m$ For each $i$, there exists $j_{0}>i+m$ with the property that each $j>j_{0}$ admits $i_{0}>f(j), i$ such that, for each $i^{\prime}>i_{0}$,

$$
p_{i i^{\prime}}^{-1} \mathcal{U}_{i}<p_{f(j), i^{\prime}}^{-1} f_{j}^{-1} q_{i+m, j}^{-1} \mathcal{V}_{i+m}
$$

Then the following implications hold for $m \in \mathbb{Z}$ :

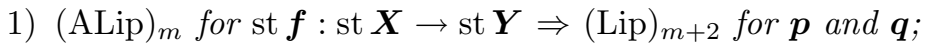

2) If all $p_{i}$ are surjective, (Lip) $)_{m}$ for $\boldsymbol{p}$ and $\boldsymbol{q} \Rightarrow(\text { ALip) })_{m+4}$ for st $\boldsymbol{f}$ : st $\boldsymbol{X} \rightarrow$ st $\boldsymbol{Y}$.

REMARK 3.6. Propety (ALip) ${ }_{-m}(m<0)$ was called property $(\text { ACon })_{m}$ in $[9]$.

Theorems 3.2 and 3.4 imply

COROLlary 3.7. Under the same setting as in Theorem 3.1, a map $f$ : $X \rightarrow Y$ is a $(\mathbb{U}, \mathbb{V})$-biLipschitz map if and only if there exists $m \geq 0$ for which $(N)_{m, 0}$ and $(N)^{m, 0}$ hold.

Theorems 3.3 and 3.5 imply

COROLlary 3.8. Under the same setting as in Theorem 3.3, a map $f: X \rightarrow Y$ is a $(\boldsymbol{p}, \boldsymbol{q})$-biLipschitz map if and only if for any approximate resolution $(\boldsymbol{f}, \boldsymbol{p}, \boldsymbol{q})$ of $f$ where $\boldsymbol{p}: X \rightarrow \boldsymbol{X}$ and $\boldsymbol{q}: Y \rightarrow \boldsymbol{Y}$ are admissible approximate resolutions of $X$ and $Y$, respectively, with each $p_{i}$ being surjective, there exist $m, n \in \mathbb{Z}$ for which (ALip) $_{m}$ and (ALip) ${ }^{n}$ hold for st $\boldsymbol{f}:$ st $\boldsymbol{X} \rightarrow$ st $\boldsymbol{Y}$.

We will need another characterization for condition (ALip) $)_{m}$. For each approximate map $\boldsymbol{f}=\left\{f_{j}, f\right\}: \boldsymbol{X} \rightarrow \boldsymbol{Y}$ where $\boldsymbol{p}=\left\{p_{i}\right\}: X \rightarrow \boldsymbol{X}=$ $\left\{X_{i}, \mathcal{U}_{i}, p_{i i^{\prime}}\right\}$ and $\boldsymbol{q}=\left\{q_{j}\right\}: Y \rightarrow \boldsymbol{Y}=\left\{Y_{j}, \mathcal{V}_{j}, q_{j j^{\prime}}\right\}$ are admissible approximate resolutions, consider the following properties for $m \geq 0$ :

$(\text { ALip })_{m}^{*}$ For each $i$ there exists $i_{0}>f(i), i+m$ such that, for each $i^{\prime}>i_{0}$,

$$
p_{i+m, i^{\prime}}^{-1} \mathcal{U}_{i+m}<p_{f(i), i^{\prime}}^{-1} f_{i}^{-1} \mathcal{V}_{i}
$$

and

$(\mathrm{ALip})_{-m}^{*}$ For each $i$ there exists $i_{0}>f(i+m)$ such that, for each $i^{\prime}>i_{0}$,

$$
p_{i i^{\prime}}^{-1} \mathcal{U}_{i+m}<p_{f(i+m), i^{\prime}}^{-1} f_{i+m}^{-1} \mathcal{V}_{i+m}
$$

THEOREM 3.9. The following implications hold for $m \in \mathbb{Z}$ :

1) $(\mathrm{ALip})_{m}$ for st $\boldsymbol{f}:$ st $\boldsymbol{X} \rightarrow$ st $\boldsymbol{Y} \Rightarrow(\mathrm{ALip})_{m}^{*}$ for $\boldsymbol{i}_{\mathrm{st} \boldsymbol{Y}}$ st $\boldsymbol{f}:$ st $\boldsymbol{X} \rightarrow$ $\mathrm{st}^{2} \boldsymbol{Y}$

2) $\left(\right.$ ALip) ${ }_{m}^{*}$ for st $\boldsymbol{f}:$ st $\boldsymbol{X} \rightarrow$ st $\boldsymbol{Y} \Rightarrow(\text { ALip })_{m}$ for $\boldsymbol{i}_{\text {st } \boldsymbol{Y}}$ st $\boldsymbol{f}:$ st $\boldsymbol{X} \rightarrow$ st $^{2} \boldsymbol{Y}$

3) $(\text { ALip })_{m}$ for $\boldsymbol{i}_{\text {st } \boldsymbol{Y}}$ st $\boldsymbol{f}:$ st $\boldsymbol{X} \rightarrow \mathrm{st}^{2} \boldsymbol{Y} \Rightarrow(\mathrm{ALip})_{m+1}$ for $\mathrm{st}^{2} \boldsymbol{f}$ : $\mathrm{st}^{2} \boldsymbol{X} \rightarrow \mathrm{st}^{2} \boldsymbol{Y}$ 
4) $\left(\right.$ ALip) ${ }_{m}^{*}$ for $\boldsymbol{i}_{\mathrm{st} \boldsymbol{Y}}$ st $\boldsymbol{f}:$ st $\boldsymbol{X} \rightarrow \mathrm{st}^{2} \boldsymbol{Y} \Rightarrow(\mathrm{ALip})_{m+1}^{*}$ for $\mathrm{st}^{2} \boldsymbol{f}$ : $\mathrm{st}^{2} \boldsymbol{X} \rightarrow \mathrm{st}^{2} \boldsymbol{Y}$

5) (ALip) $)_{m}$ for st $\boldsymbol{f}:$ st $\boldsymbol{X} \rightarrow$ st $\boldsymbol{Y} \Rightarrow(\text { ALip })_{m+1}^{*}$ for $\mathrm{st}^{2} \boldsymbol{f}: \mathrm{st}^{2} \boldsymbol{X} \rightarrow$ $\mathrm{st}^{2} \boldsymbol{Y}$

6) $\left(\right.$ ALip) ${ }_{m}^{*}$ for st $\boldsymbol{f}:$ st $\boldsymbol{X} \rightarrow$ st $\boldsymbol{Y} \Rightarrow(\text { ALip })_{m+1}$ for $\mathrm{st}^{2} \boldsymbol{f}: \mathrm{st}^{2} \boldsymbol{X} \rightarrow$ $\mathrm{st}^{2} \boldsymbol{Y}$.

Proof. Throughout the proof, assume $m \geq 0$. The argument for $m<0$ is similar. To see 1 ), let $i \in \mathbb{N}$. Take $j_{0}>i$ as in (ALip) $)_{m}$. Fix $j>j_{0}$. Then, by (ALip) $m$ and (AM), there exists $i_{0}>f(j), i+m$ such that for each $i^{\prime}>i_{0}$,

$$
p_{i+m, i^{\prime}}^{-1} \operatorname{st}_{\mathcal{U}_{i+m}}<p_{f(j), i^{\prime}}^{-1} f_{j}^{-1} q_{i j}^{-1} \text { st } \mathcal{V}_{i},
$$

and

$$
\left(f_{i} p_{f(i), i^{\prime}}, q_{i j} f_{j} p_{f(j), i^{\prime}}\right)<\operatorname{st} \mathcal{V}_{i}
$$

By $(3.8)$,

$$
p_{f(j), i^{\prime}}^{-1} f_{j}^{-1} q_{i j}^{-1} \text { st } \mathcal{V}_{i}<p_{f(i), i^{\prime}}^{-1} f_{i}^{-1} \mathrm{st}^{2} \mathcal{V}_{i}
$$

By (3.7) and (3.9),

$$
p_{i+m, i^{\prime}}^{-1} \mathrm{st}_{\mathcal{U}_{i+m}}<p_{f(i), i^{\prime}}^{-1} f_{i}^{-1} \mathrm{st}^{2} \mathcal{V}_{i} \text { for } i^{\prime}>i_{0},
$$

proving 1 ). To see 2 ), let $i \in \mathbb{N}$, and let $j_{0}=i+1$. Then by (ALip) ${ }_{m}^{*}$ and (AM) for $\boldsymbol{f}: \boldsymbol{X} \rightarrow \boldsymbol{Y}$, for each $j>j_{0}$, there exists $i_{0}>f(j), i+m$ such that for each $i^{\prime}>i_{0}$,

$$
p_{i+m, i^{\prime}}^{-1} \operatorname{st} \mathcal{U}_{i+m}<p_{f(i), i^{\prime}}^{-1} f_{i}^{-1} \text { st } \mathcal{V}_{i}
$$

and

$$
\left(f_{i} p_{f(i), i^{\prime}}, q_{i j} f_{j} p_{f(j), i^{\prime}}\right)<\operatorname{st} \mathcal{V}_{i}
$$

By (3.11),

$$
p_{f(i), i^{\prime}}^{-1} f_{i}^{-1} \mathrm{st} \mathcal{V}_{i}<p_{f(j), i^{\prime}}^{-1} f_{j}^{-1} q_{i j}^{-1} \mathrm{st}^{2} \mathcal{V}_{i}
$$

By (3.10) and (3.12),

$$
p_{i+m, i^{\prime}}^{-1} \operatorname{st} \mathcal{U}_{i+m}<p_{f(j), i^{\prime}}^{-1} f_{j}^{-1} q_{i j}^{-1} \mathrm{st}^{2} \mathcal{V}_{i}
$$

verifying 2). To see 3 ), let $i \in \mathbb{N}$. Then the hypothesis together with (A1) implies that there exists $j_{0}>i$ with the property that each $j>j_{0}$ admits $i_{0}>f(j), i+m+1$ such that for each $i^{\prime}>i_{0}$,

$$
p_{i+m, i^{\prime}}^{-1} \mathrm{st}_{i+m}<p_{f(j), i^{\prime}}^{-1} f_{j}^{-1} q_{i j}^{-1} \mathrm{st}^{2} \mathcal{V}_{i}
$$

and

$$
\left(p_{i+m, i^{\prime}}, p_{i+m, i+m+1} p_{i+m+1, i^{\prime}}\right)<\mathcal{U}_{i+m} .
$$

By (3.14) and (U),

$$
p_{i+m+1, i^{\prime}}^{-1} \mathrm{st}^{2} \mathcal{U}_{i+m+1}<p_{i+m+1, i^{\prime}}^{-1} p_{i+m, i+m+1}^{-1} \mathcal{U}_{i+m}<p_{i+m, i^{\prime}}^{-1} \text { st } \mathcal{U}_{i+m}
$$


By (3.15) and (3.13),

$$
p_{i+m+1, i^{\prime}}^{-1} \mathrm{st}^{2} \mathcal{U}_{i+m+1}<p_{f(j), i^{\prime}}^{-1} f_{j}^{-1} q_{i j}^{-1} \mathrm{st}^{2} \mathcal{V}_{i} \text { for } i^{\prime}>i_{0},
$$

proving 3). 4) is similar to 3), and 5) and 6) easily follow from 1),4) and 2), $3)$, respectively.

We will also need another characterization for property $(\text { ALip })^{m}$. For each approximate map $\boldsymbol{f}=\left\{f_{j}, f\right\}: \boldsymbol{X} \rightarrow \boldsymbol{Y}$ where $\boldsymbol{p}=\left\{p_{i}\right\}: X \rightarrow \boldsymbol{X}=$ $\left\{X_{i}, \mathcal{U}_{i}, p_{i i^{\prime}}\right\}$ and $\boldsymbol{q}=\left\{q_{j}\right\}: Y \rightarrow \boldsymbol{Y}=\left\{Y_{j}, \mathcal{V}_{j}, q_{j j^{\prime}}\right\}$ are approximate resolutions of $X$ and $Y$, respectively, for $m \geq 0$ consider the following properties for $m \geq 0$ :

$(\mathrm{ALip})_{*}^{m}$ For each $i$, there exists $i_{0}>f(i), i+m$ such that for each $i^{\prime}>i_{0}$,

$$
p_{f(i), i^{\prime}}^{-1} f_{i}^{-1} \mathcal{V}_{i}<p_{i+m, i^{\prime}}^{-1} \mathcal{U}_{i+m}
$$

and

(ALip) $)_{*}^{-m}$ For each $i$, there exists $i_{0}>f(i+m)$ such that for each $i^{\prime}>i_{0}$,

$$
p_{f(i+m), i^{\prime}}^{-1} f_{i+m}^{-1} \mathcal{V}_{i+m}<p_{i i^{\prime}}^{-1} \mathcal{U}_{i} \text {. }
$$

THEOREM 3.10. The following implications hold for $m \in \mathbb{Z}$ :

1) $(\text { ALip) })^{m}$ for $\mathrm{st}^{3} \boldsymbol{f}: \mathrm{st}^{3} \boldsymbol{X} \rightarrow \mathrm{st}^{3} \boldsymbol{Y} \Rightarrow$ (ALip) ${ }_{*}^{m-1}$ for $\mathrm{st}^{2} \boldsymbol{f}: \mathrm{st}^{2} \boldsymbol{X} \rightarrow$ $\mathrm{st}^{2} \boldsymbol{Y}$; and

2) (ALip) ${ }_{*}^{m}$ for $\mathrm{st}^{3} \boldsymbol{f}: \mathrm{st}^{3} \boldsymbol{X} \rightarrow \mathrm{st}^{3} \boldsymbol{Y} \Rightarrow\left(\right.$ ALip) ${ }^{m-1}$ for $\mathrm{st}^{2} \boldsymbol{f}: \mathrm{st}^{2} \boldsymbol{X} \rightarrow$ $\mathrm{st}^{2} \boldsymbol{Y}$.

Proof. Assume $m \geq 1$ since the argument for the case $m \leq 0$ is similar. For 1), let $i \in \mathbb{N}$. By (ALip) ${ }^{m}$ for $\mathrm{st}^{3} \boldsymbol{f}: \mathrm{st}^{3} \boldsymbol{X} \rightarrow \mathrm{st}^{3} \boldsymbol{Y}$ and (AM) for $\boldsymbol{f}: \boldsymbol{X} \rightarrow \boldsymbol{Y}$, there exist $j>i$ and $i_{0}$ with $i_{0}>i+m, f(j)$ such that for $i^{\prime}>i_{0}$

$$
p_{f(j), i^{\prime}}^{-1} f_{j}^{-1} q_{i j}^{-1} \mathrm{st}^{3} \mathcal{V}_{i}<p_{i+m, i^{\prime}}^{-1} \mathrm{st}^{3} \mathcal{U}_{i+m}
$$

and

$$
\left(f_{i} p_{f(i), i^{\prime}}, q_{i j} f_{j} p_{f(j), i^{\prime}}\right)<\operatorname{st} \mathcal{V}_{i}
$$

By (3.17) and (3.16),

$$
p_{f(i), i^{\prime}}^{-1} f_{i}^{-1} \mathrm{st}^{2} \mathcal{V}_{i}<p_{i+m, i^{\prime}}^{-1} \mathrm{st}^{3} \mathcal{U}_{i+m} \text { for } i^{\prime}>i_{0} .
$$

But since, by (A1),

$$
\left(p_{i+m-1, i+m} p_{i+m, i^{\prime}}, p_{i+m-1, i^{\prime}}\right)<\mathcal{U}_{i+m-1},
$$

then, by $(\mathrm{U})$, for $i^{\prime}>i_{0}$,

$$
\begin{aligned}
p_{i+m, i^{\prime}}^{-1} \mathrm{st}^{3} \mathcal{U}_{i+m} & <p_{i+m, i^{\prime}}^{-1} \text { st } p_{i+m-1, i+m}^{-1} \mathcal{U}_{i+m-1} \\
& <p_{i+m, i^{\prime}}^{-1} p_{i+m-1, i+m}^{-1} \mathrm{st} \mathcal{U}_{i+m-1} \\
& <p_{i+m-1, i^{\prime}}^{-1} \mathrm{st}^{2} \mathcal{U}_{i+m-1}
\end{aligned}
$$


This together with (3.18) implies

$$
p_{f(j), i^{\prime}}^{-1} f_{i}^{-1} \mathrm{st}^{2} \mathcal{V}_{i}<p_{i+m-1, i^{\prime}}^{-1} \mathrm{st}^{2} \mathcal{U}_{i+m-1} \text { for } i^{\prime}>i_{0} .
$$

This means (ALip) ${ }_{*}^{m-1}$ for $\mathrm{st}^{2} \boldsymbol{f}: \mathrm{st}^{2} \boldsymbol{X} \rightarrow \mathrm{st}^{2} \boldsymbol{Y}$.

For 2), let $i \in \mathbb{N}$, and let $j_{0}=i$. Fix $j>j_{0}$. By (ALip) ${ }_{*}^{m}$ for $\mathrm{st}^{3} \boldsymbol{f}$ : $\mathrm{st}^{3} \boldsymbol{X} \rightarrow \mathrm{st}^{3} \boldsymbol{Y}$, (LAM) and (A1), there is $i_{0}>i+m, f(j)$ with the property that for each $i^{\prime}>i_{0}$,

$$
p_{f(i), i^{\prime}}^{-1} f_{i}^{-1} \mathrm{st}^{3} \mathcal{V}_{i}<p_{i+m, i^{\prime}}^{-1} \mathrm{st}^{3} \mathcal{U}_{i+m}
$$

and (3.17) and (3.19) hold. By (3.17), (3.20) and (3.19), for each $i^{\prime}>i_{0}$,

$$
p_{f(j), i^{\prime}}^{-1} f_{j}^{-1} q_{i j}^{-1} \mathrm{st}^{2} \mathcal{V}_{i}<p_{i+m-1, i^{\prime}}^{-1} \mathrm{st}^{2} \mathcal{U}_{i+m-1}
$$

which means (ALip) ${ }^{m-1}$ for $\mathrm{st}^{2} \boldsymbol{f}: \mathrm{st}^{2} \boldsymbol{X} \rightarrow \mathrm{st}^{2} \boldsymbol{Y}$.

\section{The BOX-COUNTING DIMENSION IS LipSCHITZ INVARIANT}

Let $X$ be any space. For each $\mathcal{U} \in \operatorname{Cov}(X)$, let

$$
N_{\mathcal{U}}(X)=\min \left\{n: X \subseteq U_{1} \cup \cdots \cup U_{n}, U_{i} \in \mathcal{U}\right\} .
$$

For each normal sequence $\mathbb{U}=\left\{\mathcal{U}_{i}\right\}$ on a space $X$, we respectively define the lower and the upper box-counting dimensions of $(X, \mathbb{U})$ by

$$
\underline{\operatorname{dim}}_{B}(X, \mathbb{U})=\varliminf_{i \rightarrow \infty} \frac{\log _{3} N_{\mathcal{U}_{i}}(X)}{i}
$$

and

$$
\overline{\operatorname{dim}}_{B}(X, \mathbb{U})=\varlimsup_{i \rightarrow \infty} \frac{\log _{3} N_{\mathcal{U}_{i}}(X)}{i} .
$$

If the two values coincide, the common value is called the box-counting dimension of $(X, \mathbb{U})$ and is denoted by $\operatorname{dim}_{B}(X, \mathbb{U})$.

If $X$ is a compact subset of $\mathbb{R}^{m}$ with the usual metric and if we take the normal sequence $\mathbb{U}$ of open coverings $\mathcal{U}_{i}$ by open balls with radius $\frac{1}{3^{i}}$, then the above values coincide with the usual box-counting dimension.

The fundamental properties of the box-counting dimension for normal sequences can be found in [9]. In particular, we have the following Lipschitz subinvariance property for the box-counting dimension.

TheOREM 4.1. Let $\mathbb{U}=\left\{\mathcal{U}_{i}\right\}$ and $\mathbb{V}=\left\{\mathcal{V}_{i}\right\}$ be normal sequences on $X$ and $Y$, respectively. If $f: X \rightarrow Y$ is a surjective $(\mathbb{U}, \mathbb{V})$-Lipschitz map, then

$$
\underline{\operatorname{dim}}_{B}(Y, \mathbb{V}) \leq \underline{\operatorname{dim}}_{B}(X, \mathbb{U})
$$

and

$$
\overline{\operatorname{dim}}_{B}(Y, \mathbb{V}) \leq \overline{\operatorname{dim}}_{B}(X, \mathbb{U}) .
$$

Proof. See [9, Proposition 4.7].

Now we have the following Lipschitz invariance property: 
TheOREM 4.2. Let $\mathbb{U}=\left\{\mathcal{U}_{i}\right\}$ and $\mathbb{V}=\left\{\mathcal{V}_{i}\right\}$ be normal sequences on $X$ and $Y$, respectively. If $f: X \rightarrow Y$ is a surjective $(\mathbb{U}, \mathbb{V})$-biLipschitz map, then

$$
\underline{\operatorname{dim}}_{B}(Y, \mathbb{V})=\underline{\operatorname{dim}}_{B}(X, \mathbb{U})
$$

and

$$
\overline{\operatorname{dim}}_{B}(Y, \mathbb{V})=\overline{\operatorname{dim}}_{B}(X, \mathbb{U}) .
$$

Proof. By Theorem 4.1, it suffices to show that

$$
\underline{\operatorname{dim}}_{B}(X, \mathbb{U}) \leq \underline{\operatorname{dim}}_{B}(Y, \mathbb{V}) \text { and } \overline{\operatorname{dim}}_{B}(X, \mathbb{U}) \leq \overline{\operatorname{dim}}_{B}(Y, \mathbb{V}) .
$$

By Theorem 3.2, $f^{-1} \Sigma^{m} \mathbb{V}<\mathbb{U}$, for some $m \geq 0$. Then for each $j \geq 1$,

$$
N_{\mathcal{V}_{j+m}}(Y) \geq N_{f^{-1}} \mathcal{V}_{j+m}(X) \geq N_{\mathcal{U}_{j}}(X) .
$$

This easily implies (4.1).

Let $\boldsymbol{p}=\left\{p_{i}\right\}: X \rightarrow \boldsymbol{X}=\left\{X_{i}, \mathcal{U}_{i}, p_{i i^{\prime}}\right\}$ be an approximate resolution. For each $i \in \mathbb{N}$, let

$$
\beta_{i}(\boldsymbol{X})=\varlimsup_{j \rightarrow \infty} N_{p_{i j}^{-1} \mathcal{U}_{i}}\left(X_{j}\right) .
$$

Then we define the upper and the lower box-counting dimensions of $\boldsymbol{p}: X \rightarrow$ $\boldsymbol{X}$ respectively by

$$
\overline{\operatorname{dim}}_{B}(\boldsymbol{p}: X \rightarrow \boldsymbol{X})=\varlimsup_{i \rightarrow \infty} \frac{\log _{3} \beta_{i}(\boldsymbol{X})}{i}
$$

and

$$
\underline{\operatorname{dim}}_{B}(\boldsymbol{p}: X \rightarrow \boldsymbol{X})=\varliminf_{i \rightarrow \infty} \frac{\log _{3} \beta_{i}(\boldsymbol{X})}{i} .
$$

If the two values coincide, then we write $\operatorname{dim}_{B}(\boldsymbol{p}: X \rightarrow \boldsymbol{X})$ for the common value and call it the box-counting dimension of $\boldsymbol{p}: X \rightarrow \boldsymbol{X}$. Note that by $[9$, Proposition 5.5], for $m \geq 1$,

$$
\overline{\operatorname{dim}}_{B}(\operatorname{st} \boldsymbol{p}: X \rightarrow \operatorname{st} \boldsymbol{X})=\overline{\operatorname{dim}}_{B}\left(\mathrm{st}^{m} \boldsymbol{p}: X \rightarrow \mathrm{st}^{m} \boldsymbol{X}\right),
$$

and

$$
\underline{\operatorname{dim}}_{B}(\operatorname{st} \boldsymbol{p}: X \rightarrow \operatorname{st} \boldsymbol{X})=\underline{\operatorname{dim}}_{B}\left(\mathrm{st}^{m} \boldsymbol{p}: X \rightarrow \mathrm{st}^{m} \boldsymbol{X}\right) .
$$

Hence, we define

$$
\overline{\operatorname{Dim}}_{B}(\boldsymbol{p}: X \rightarrow \boldsymbol{X})=\overline{\operatorname{dim}}_{B}(\operatorname{st} \boldsymbol{p}: X \rightarrow \text { st } \boldsymbol{X}),
$$

and

$$
\underline{\operatorname{Dim}}_{B}(\boldsymbol{p}: X \rightarrow \boldsymbol{X})=\underline{\operatorname{dim}}_{B}(\text { st } \boldsymbol{p}: X \rightarrow \text { st } \boldsymbol{X}) .
$$

The fundamental properties of the box-counting dimension for approximate resolutions can be found in [9]. In particular, we have the following Lipschitz subinvariance property for the box-counting dimension. 
Theorem 4.3. Let $\boldsymbol{p}=\left\{p_{i}\right\}: X \rightarrow \boldsymbol{X}=\left\{X_{i}, \mathcal{U}_{i}, p_{i i^{\prime}}\right\}$ and $\boldsymbol{q}=\left\{q_{j}\right\}$ : $Y \rightarrow \boldsymbol{Y}=\left\{Y_{j}, \mathcal{V}_{j}, q_{j j^{\prime}}\right\}$ be approximate resolutions, and let $\boldsymbol{f}=\left\{f_{j}, f\right\}$ : $\boldsymbol{X} \rightarrow \boldsymbol{Y}$ be an approximate map with property (APS). If st $\boldsymbol{f}:$ st $\boldsymbol{X} \rightarrow \operatorname{st} \boldsymbol{Y}$ satisfies (ALip) ${ }_{m}$ for some $m \geq 0$, then

$$
\underline{\operatorname{dim}}_{B}(\operatorname{st} \boldsymbol{p}: X \rightarrow \operatorname{st} \boldsymbol{X}) \geq \underline{\operatorname{dim}}_{B}(\operatorname{st} \boldsymbol{q}: Y \rightarrow \operatorname{st} \boldsymbol{Y})
$$

and

$$
\overline{\operatorname{dim}}_{B}(\operatorname{st} \boldsymbol{p}: X \rightarrow \operatorname{st} \boldsymbol{X}) \geq \overline{\operatorname{dim}}_{B}(\operatorname{st} \boldsymbol{q}: Y \rightarrow \operatorname{st} \boldsymbol{Y}) .
$$

Proof. See [9, Corollary 7.2].

Now we prove the opposite inequalities:

Theorem 4.4. Let $\boldsymbol{p}=\left\{p_{i}\right\}: X \rightarrow \boldsymbol{X}=\left\{X_{i}, \mathcal{U}_{i}, p_{i i^{\prime}}\right\}$ and $\boldsymbol{q}=\left\{q_{j}\right\}$ : $Y \rightarrow \boldsymbol{Y}=\left\{Y_{j}, \mathcal{V}_{j}, q_{j j^{\prime}}\right\}$ be approximate resolutions, and let $\boldsymbol{f}=\left\{f_{j}, f\right\}$ : $\boldsymbol{X} \rightarrow \boldsymbol{Y}$ be an approximate map. If $\boldsymbol{f}: \boldsymbol{X} \rightarrow \boldsymbol{Y}$ satisfies (ALip) ${ }^{m}$ for some $m \in \mathbb{Z}$, then

$$
\underline{\operatorname{dim}}_{B}(\boldsymbol{p}: X \rightarrow \boldsymbol{X}) \leq \underline{\operatorname{dim}}_{B}(\boldsymbol{q}: Y \rightarrow \boldsymbol{Y})
$$

and

$$
\overline{\operatorname{dim}}_{B}(\boldsymbol{p}: X \rightarrow \boldsymbol{X}) \leq \overline{\operatorname{dim}}_{B}(\boldsymbol{q}: Y \rightarrow \boldsymbol{Y}) .
$$

Proof. It suffices to prove the assertion for (ALip) ${ }^{m}$ for some $m \geq 0$, because the case for $m<0$ is similar. Let $i \in \mathbb{N}$, and take $j_{0}>i$ as in $(\text { ALip })^{m}$. Fix $j>j_{0}$. Then there exists $i_{0}>f(j), i+m$ such that for each $i^{\prime}>i_{0}$

$$
p_{f(j), i^{\prime}}^{-1} f_{j}^{-1} q_{i j}^{-1} \mathcal{V}_{i}<p_{i+m, i^{\prime}}^{-1} \mathcal{U}_{i+m}
$$

This implies that for each $i^{\prime}>i_{0}$,

$$
N_{q_{i j}^{-1} \mathcal{V}_{i}}\left(Y_{j}\right) \geq N_{p_{f(j), i^{\prime}}^{-1} f_{j}^{-1} q_{i j}^{-1} \mathcal{V}_{i}}\left(X_{i^{\prime}}\right) \geq N_{p_{i+m, i^{\prime}}^{-1} \mathcal{U}_{i+m}}\left(X_{i^{\prime}}\right) .
$$

So $N_{q_{i j}^{-1}}\left(Y_{j}\right) \geq \beta_{i+m}(\boldsymbol{X})$ for each $j>j_{0}$, and thus, $\beta_{i}(\boldsymbol{Y}) \geq \beta_{i+m}(\boldsymbol{X})$. This easily implies (4.2) and (4.3).

Theorems 4.3 and 4.4 now imply the following Lipschitz invariance property for box-counting dimension:

Corollary 4.5. Let $\boldsymbol{p}, \boldsymbol{q}$ and $\boldsymbol{f}$ be as in Theorem 4.4. If st $\boldsymbol{f}:$ st $\boldsymbol{X} \rightarrow$ st $\boldsymbol{Y}$ satisfies (ALip) ${ }_{m}$ and (ALip) ${ }^{n}$ for some $m, n \in \mathbb{Z}$, then

$$
\underline{\operatorname{dim}}_{B}(\operatorname{st} \boldsymbol{p}: X \rightarrow \operatorname{st} \boldsymbol{X})=\underline{\operatorname{dim}}_{B}(\operatorname{st} \boldsymbol{q}: Y \rightarrow \operatorname{st} \boldsymbol{Y})
$$

and

$$
\overline{\operatorname{dim}}_{B}(\operatorname{st} \boldsymbol{p}: X \rightarrow \text { st } \boldsymbol{X})=\overline{\operatorname{dim}}_{B}(\operatorname{st} \boldsymbol{q}: Y \rightarrow \operatorname{st} \boldsymbol{Y}) .
$$




\section{CAtegory Whose Morphisms ARe Lipschitz Maps}

Before considering biLipschitz maps, in this section we construct a category LIP whose morphisms are based on those approximate maps which correspond to Lipschitz maps, so that the box-counting dimension is invariant in this category.

Let the objects of LIP be all admissible approximate resolutions. We defined morphisms as follows: Let $\operatorname{UALip}(\boldsymbol{X}, \boldsymbol{Y})$ denote the set of all uniform approximate maps with properties (ALip) ${ }_{m}^{*}$ for some $m \geq 0$ and (APS).

Theorem 5.1. Let $\boldsymbol{p}=\left\{p_{i}\right\}: X \rightarrow\left\{X_{i}, \mathcal{U}_{i}, p_{i i^{\prime}}\right\}$ and $\boldsymbol{q}=\left\{q_{j}\right\}: Y \rightarrow$ $\boldsymbol{Y}=\left\{Y_{j}, \mathcal{V}_{j}, q_{j j^{\prime}}\right\}$ be admissible approximate resolutions of $X$ and $Y$, respectively. For each approximate map $\boldsymbol{f}=\left\{f_{j}, f\right\}$ : st $\boldsymbol{X} \rightarrow$ st $\boldsymbol{Y}$ with property (ALip) ${ }_{m}^{*}$ for some $m \geq 0$, there exists a uniform approximate map $\boldsymbol{f}^{\prime}=\left\{f_{j}^{\prime}, f^{\prime}\right\}: \mathrm{st}^{2} \boldsymbol{X} \rightarrow \mathrm{st}^{2} \boldsymbol{Y}$ with property (ALip) ${ }_{m+1}^{*}$ that represents the same limit map $f: X \rightarrow Y$ as $\boldsymbol{f}$.

Proof. For each $j \in \mathbb{N}$, let $f^{\prime}(j)$ be the smallest integer $i$ with the following four properties:

1) $i \geq f(j)$

2) $\left(p_{f(j), i^{\prime}} p_{i^{\prime} i^{\prime \prime}}, p_{f(j), i^{\prime \prime}}\right)<f_{j}^{-1}$ st $\mathcal{V}_{j}$ for $i^{\prime \prime}>i^{\prime} \geq i$;

3) $p_{j+m, i^{\prime}}^{-1}$ st $\mathcal{U}_{j+m}<p_{f(j), i^{\prime}}^{-1} f_{j}^{-1}$ st $\mathcal{V}_{j}$ for $i^{\prime} \geq i$; and

4) $i \geq f^{\prime}(j-1)$ if $j \geq 2$.

For each $j$, let $f_{j}^{\prime}: X_{f^{\prime}(j)} \rightarrow Y_{j}$ be defined as $f_{j}^{\prime}=f_{j} p_{f(j), f^{\prime}(j)}$.

Claim. $\boldsymbol{f}^{\prime}=\left\{f_{j}^{\prime}, f^{\prime}\right\}: \mathrm{st}^{2} \boldsymbol{X} \rightarrow \mathrm{st}^{2} \boldsymbol{Y}$ forms a uniform approximate map with property (ALip) ${ }_{m+1}^{*}$.

First, show that $\boldsymbol{f}^{\prime}$ is an approximate map, i.e., have property (AM). Let $j<j^{\prime}$. Then (AM) for $\boldsymbol{f}$ means that there exists $i_{0}>f(j), f\left(j^{\prime}\right)$ such that for each $i^{\prime}>i_{0}$,

$$
\left(f_{j} p_{f(j), i^{\prime}}, q_{j j^{\prime}} f_{j^{\prime}} p_{f\left(j^{\prime}\right), i^{\prime}}\right)<\mathrm{st} \mathcal{V}_{j}
$$

Let $i_{0}^{\prime}>f^{\prime}(j), f^{\prime}\left(j^{\prime}\right), i_{0}$. By the choices of $f^{\prime}(j)$ and $f^{\prime}\left(j^{\prime}\right)$, for $i^{\prime}>i_{0}^{\prime}$,

$$
\left(f_{j} p_{f(j), i^{\prime}}, f_{j} p_{f(j), f^{\prime}(j)} p_{f^{\prime}(j), i^{\prime}}\right)<\mathrm{st} \mathcal{V}_{j}
$$

and

$$
\left(f_{j^{\prime}} p_{f\left(j^{\prime}\right), i^{\prime}}, f_{j^{\prime}} p_{f\left(j^{\prime}\right), f^{\prime}\left(j^{\prime}\right)} p_{f^{\prime}\left(j^{\prime}\right), i^{\prime}}\right)<\text { st } \mathcal{V}_{j^{\prime}} .
$$

(5.3) and (U) imply

$$
\left(q_{j j^{\prime}} f_{j^{\prime}} p_{f\left(j^{\prime}\right), i^{\prime}}, q_{j j^{\prime}} f_{j^{\prime}} p_{f\left(j^{\prime}\right), f^{\prime}\left(j^{\prime}\right)} p_{f^{\prime}\left(j^{\prime}\right), i^{\prime}}\right)<\mathrm{st} \mathcal{V}_{j} .
$$

By (5.2), (5.1) and (5.4),

$$
\left(f_{j^{\prime}} p_{f\left(j^{\prime}\right), f^{\prime}\left(j^{\prime}\right)} p_{f^{\prime}\left(j^{\prime}\right), i^{\prime}}, q_{j j^{\prime}} f_{j^{\prime}} p_{f\left(j^{\prime}\right), f^{\prime}\left(j^{\prime}\right)} p_{f^{\prime}\left(j^{\prime}\right), i^{\prime}}\right)<\mathrm{st}^{2} \mathcal{V}_{j}
$$

which means

$$
\left(f_{j}^{\prime} p_{f^{\prime}(j), i^{\prime}}, q_{j j^{\prime}} f_{j^{\prime}}^{\prime} p_{f^{\prime}\left(j^{\prime}\right), i^{\prime}}\right)<\mathrm{st}^{2} \mathcal{V}_{j}
$$


verifying (AM) for $\boldsymbol{f}^{\prime}:$ st $\boldsymbol{X} \rightarrow \mathrm{st} \boldsymbol{Y}$ and thus, for $\boldsymbol{f}^{\prime}: \mathrm{st}^{2} \boldsymbol{X} \rightarrow \mathrm{st}^{2} \boldsymbol{Y}$. The approximate map $\boldsymbol{f}^{\prime}: \mathrm{st}^{2} \boldsymbol{X} \rightarrow \mathrm{st}^{2} \boldsymbol{Y}$ is uniform since, by (U) and 3), for each $j$,

$$
\mathrm{st}^{2} \mathcal{U}_{f^{\prime}(j)}<p_{j+m, f^{\prime}(j)}^{-1} \mathrm{st}_{\mathcal{U}_{j+m}}<f_{j}^{\prime-1} \mathrm{st} \mathcal{V}_{j}<f_{j}^{\prime-1} \mathrm{st}^{2} \mathcal{V}_{j}
$$

It remains to verify (ALip) ${ }_{m+1}^{*}$. Indeed, by 3 ), for each $i^{\prime}>f^{\prime}(j), j+m+1$,

$$
p_{j+m, i^{\prime}}^{-1} \operatorname{st} \mathcal{U}_{j+m}<p_{f(j), i^{\prime}}^{-1} f_{j}^{-1} \text { st } \mathcal{V}_{j}
$$

But by (A1) and (U),

$$
\left(p_{j+m, i^{\prime}}, p_{j+m, j+m+1} p_{j+m+1, i^{\prime}}\right)<\mathcal{U}_{j+m},
$$

so

$$
p_{j+m+1, i^{\prime}}^{-1} \mathrm{st}^{2} \mathcal{U}_{j+m+1}<p_{j+m+1, i^{\prime}}^{-1} p_{j+m, j+m+1}^{-1} \mathcal{U}_{j+m}<p_{j+m, i^{\prime}}^{-1} \mathrm{st} \mathcal{U}_{j+m}
$$

By 2),

$$
p_{f(j), i^{\prime}}^{-1} f_{j}^{-1} \mathrm{st} \mathcal{V}_{j}<p_{f^{\prime}(j), i^{\prime}}^{-1} p_{f^{\prime}(j), f(j)}^{-1} f_{j}^{-1} \mathrm{st}^{2} \mathcal{V}_{j}
$$

By (5.7), (5.6) and (5.5),

$$
p_{j+m+1, i^{\prime}}^{-1} \mathrm{st}^{2} \mathcal{U}_{j+m+1}<p_{f^{\prime}(j), i^{\prime}}^{-1} f_{j}^{\prime-1} \mathrm{st} \mathcal{V}_{j}
$$

verifying property (ALip) ${ }_{m+1}^{*}$ for $\boldsymbol{f}^{\prime}: \mathrm{st}^{2} \boldsymbol{X} \rightarrow \mathrm{st}^{2} \boldsymbol{Y}$. This proves the claim.

It is easy to see that $\boldsymbol{f}$ and $\boldsymbol{f}^{\prime}$ induce the same limit map $f: X \rightarrow Y$. Hence $f^{\prime}$ is the desired map.

REMARK 5.2. In Theorem 5.1, if $\boldsymbol{f}$ has property (APS), $\boldsymbol{f}^{\prime}$ also has property (APS).

Theorem 5.3. Let $\boldsymbol{f}: \boldsymbol{X} \rightarrow \boldsymbol{Y}$ be an approximate map. Then if $\mathrm{st} \boldsymbol{f}:$ st $\boldsymbol{X} \rightarrow$ st $\boldsymbol{Y}$ has property (ALip) ${ }_{m}^{*}$ for some $m \geq 0$, then $\mathrm{st}^{2} \boldsymbol{f}: \mathrm{st}^{2} \boldsymbol{X} \rightarrow$ $\mathrm{st}^{2} \boldsymbol{Y}$ has property (ALip) ${ }_{m+1}^{*}$.

Proof. Let $i \in \mathbb{N}$. Then by (ALip) ${ }_{m}^{*}$ and (A1), there exists $i_{0}>i+m+$ $1, f(i)$ such that for each $i^{\prime}>i_{0}$,

$$
p_{i+m, i^{\prime}}^{-1} \operatorname{st} \mathcal{U}_{i+m}<p_{f(i), i^{\prime}}^{-1} f_{i}^{-1} \text { st } \mathcal{V}_{i}
$$

and

$$
\left(p_{i+m, i^{\prime}}, p_{i+m, i+m+1} p_{i+m+1, i^{\prime}}\right)<\mathcal{U}_{i+m} .
$$

Then, by (U), (5.9) and (5.8), for $i^{\prime}>i_{0}$,

$$
\begin{aligned}
p_{i+m+1, i^{\prime}}^{-1} \mathrm{st}^{2} \mathcal{U}_{i+m+1} & <p_{i+m+1, i^{\prime}}^{-1} p_{i+m, i+m+1}^{-1} \mathcal{U}_{i+m} \\
& <p_{i+m, i^{\prime}}^{-1} \operatorname{st} \mathcal{U}_{i+m}<p_{f(i) i^{\prime}}^{-1} f_{i}^{-1} \mathrm{st} \mathcal{V}_{i}<p_{f(i) i^{\prime}}^{-1} f_{i}^{-1} \mathrm{st}^{2} \mathcal{V}_{i}
\end{aligned}
$$

as required. 
By Theorem 5.3, there is a well-defined direct sequence:

UALip $($ st $\boldsymbol{X}$, st $\boldsymbol{Y}) \rightarrow \operatorname{UALip}\left(\mathrm{st}^{2} \boldsymbol{X}, \mathrm{st}^{2} \boldsymbol{Y}\right) \cdots \rightarrow \operatorname{UALip}\left(\mathrm{st}^{n} \boldsymbol{X}, \mathrm{st}^{n} \boldsymbol{Y}\right) \rightarrow \cdots$.

Let UALip* $(\boldsymbol{X}, \boldsymbol{Y})$ denote the direct limit of this sequence. For each admissible approximate resolutions $\boldsymbol{p}: X \rightarrow \boldsymbol{X}$ and $\boldsymbol{q}: Y \rightarrow \boldsymbol{Y}$, let the set $\operatorname{LIP}(\boldsymbol{p}, \boldsymbol{q})$ of morphisms from $\boldsymbol{p}$ to $\boldsymbol{q}$ be the set $\operatorname{UALip}^{*}(\boldsymbol{X}, \boldsymbol{Y})$.

We wish to define the composition as follows: Let $\boldsymbol{f}=\left\{f_{j}, f\right\}: \boldsymbol{X} \rightarrow \boldsymbol{Y}$ and $\boldsymbol{g}=\left\{g_{k}, g\right\}: \boldsymbol{Y} \rightarrow \boldsymbol{Z}$ be uniform approximate maps, where $\boldsymbol{p}=\left\{p_{i}\right\}$ : $X \rightarrow \boldsymbol{X}=\left\{X_{i}, \mathcal{U}_{i}, p_{i i^{\prime}}\right\}, \boldsymbol{q}=\left\{q_{j}\right\}: Y \rightarrow \boldsymbol{Y}=\left\{Y_{j}, \mathcal{V}_{j}, q_{j j^{\prime}}\right\}$ and $\boldsymbol{r}=\left\{r_{k}\right\}:$ $Z \rightarrow Z=\left\{Z_{k}, \mathcal{W}_{k}, r_{k k^{\prime}}\right\}$ are admissible approximate resolutions. Define $h=f g: \mathbb{N} \rightarrow \mathbb{N}$ and for each $k$, define $h_{k}=g_{k} f_{g(k)}: X_{f g(k)} \rightarrow Y_{k}$.

TheOREM 5.4. $\boldsymbol{h}=\left\{h_{k}, h\right\}:$ st $\boldsymbol{X} \rightarrow$ st $\boldsymbol{Z}$ is a uniform approximate map.

Proof. Let $k<k^{\prime}$. Then (AM) for $\boldsymbol{g}: \boldsymbol{Y} \rightarrow \boldsymbol{Z}$ implies that there exists $j>g(k), g\left(k^{\prime}\right)$ such that

$$
\left(g_{k} q_{g(k), j}, r_{k k^{\prime}} g_{k^{\prime}} q_{g\left(k^{\prime}\right), j}\right)<\mathrm{st} \mathcal{W}_{k} .
$$

$(\mathrm{AM})$ for $\boldsymbol{f}: \boldsymbol{X} \rightarrow \boldsymbol{Y}$ implies that there exists $i_{0}>f g(k), f g\left(k^{\prime}\right), f(j)$ such that for $i>i_{0}$,

$$
\left(f_{g(k)} p_{f g(k), i}, q_{g(k), j} f_{j} p_{f(j), i}\right)<\mathrm{st} \mathcal{V}_{g(k)}
$$

and

$$
\left(f_{g\left(k^{\prime}\right)} p_{f g\left(k^{\prime}\right), i}, q_{g\left(k^{\prime}\right), j} f_{j} p_{f(j), i}\right)<\mathrm{st} \mathcal{V}_{g\left(k^{\prime}\right)}
$$

Since $\boldsymbol{g}$ is uniform,

$$
\mathcal{V}_{g(k)}<g_{k}^{-1} \mathcal{W}_{k}
$$

and

$$
\mathcal{V}_{g\left(k^{\prime}\right)}<g_{k^{\prime}}^{-1} \mathcal{W}_{k^{\prime}}
$$

By (5.11), (5.13), (5.10), (5.12) and (5.14),

$$
\left(g_{k} f_{g(k)} p_{f g(k), i}, r_{k k^{\prime}} g_{k^{\prime}} f_{g\left(k^{\prime}\right)} p_{f g\left(k^{\prime}\right), i}\right)<\mathrm{st}^{2} \mathcal{W}_{k},
$$

proving (AM) for $\boldsymbol{h}:$ st $\boldsymbol{X} \rightarrow$ st $\boldsymbol{Z}$.

THEOREM 5.5. If st $\boldsymbol{f}:$ st $\boldsymbol{X} \rightarrow$ st $\boldsymbol{Y}$ has property (ALip) ${ }_{m}^{*}$ and if $\mathrm{st}^{2} \boldsymbol{g}:$ $\mathrm{st}^{2} \boldsymbol{Y} \rightarrow \mathrm{st}^{2} \boldsymbol{Z}$ has property (ALip) ${ }_{n}^{*}$ for some $m, n \geq 0$, then $\mathrm{st}^{2} \boldsymbol{h}: \mathrm{st}^{3} \boldsymbol{X} \rightarrow$ $\mathrm{st}^{3} \boldsymbol{Z}$ has property (ALip) ${ }_{m+n+2}^{*}$.

Proof. Let $k \in \mathbb{N}$. By $(\mathrm{ALip})_{m}^{*}$ for $\mathrm{st}^{2} \boldsymbol{g}: \mathrm{st}^{2} \boldsymbol{Y} \rightarrow \mathrm{st}^{2} \boldsymbol{Z}$, there exists $j_{0}>g(k)$ such that for $j>j_{0}$,

$$
q_{k+n, j}^{-1} \mathrm{st}^{2} \mathcal{V}_{j+n}<q_{g(k), j}^{-1} g_{k}^{-1} \mathrm{st}^{2} \mathcal{W}_{k}
$$

By $\left(\right.$ ALip) ${ }_{n}^{*}$ for st $\boldsymbol{f}:$ st $\boldsymbol{X} \rightarrow$ st $\boldsymbol{Y}$ and $(\mathrm{AM})$ for $\boldsymbol{f}$, there exists $i_{0}>k+n+$ $m, f(j+n), f g(k), f(j)$ such that for $i>i_{0}$,

$$
p_{k+n+m, i}^{-1} \text { st } \mathcal{U}_{k+n+m}<p_{f(k+n), i}^{-1} f_{k+n}^{-1} \text { st } \mathcal{V}_{k+n}
$$




$$
\left(f_{k+n} p_{f(k+n), i}, q_{k+n, j} f_{j} p_{f(j), i}\right)<\operatorname{st} \mathcal{V}_{k+n}
$$

and

$$
\left(f_{g(k)} p_{f g(k), i}, q_{g(k), j} f_{j} p_{f(j), i}\right)<\operatorname{st} \mathcal{V}_{g(k)} .
$$

By (5.17),

$$
p_{f(k+n), i}^{-1} f_{k+n}^{-1} \text { st } \mathcal{V}_{k+n}<p_{f(j), i}^{-1} f_{j}^{-1} q_{k+n, j}^{-1} \mathrm{st}^{2} \mathcal{V}_{k+n}
$$

By (5.19) and (5.15),

$$
p_{f(k+n), i}^{-1} f_{k+n}^{-1} \text { st } \mathcal{V}_{k+n}<p_{f(j), i}^{-1} f_{j}^{-1} q_{g(k), j}^{-1} g_{k}^{-1} \mathrm{st}^{2} \mathcal{W}_{k} .
$$

(5.18) and the fact that $\boldsymbol{g}$ is uniform imply that

$$
p_{f(j), i}^{-1} f_{j}^{-1} q_{g(k), j}^{-1} g_{k}^{-1} \mathrm{st}^{2} \mathcal{W}_{k}<p_{f(k), i}^{-1} f_{g(k)}^{-1} g_{k}^{-1} \mathrm{st}^{3} \mathcal{W}_{k}
$$

By (5.16), (5.20), (5.21),

$$
p_{k+n+m, i}^{-1} \operatorname{st} \mathcal{U}_{k+n+m}<p_{f g(k), i}^{-1} f_{g(k)}^{-1} g_{k}^{-1} \mathrm{st}^{3} \mathcal{W}_{k} .
$$

This means (ALip) ${ }_{m+n}^{*}$ for $\boldsymbol{i}_{\mathrm{st}^{2}} \boldsymbol{Z} \boldsymbol{i}_{\mathrm{st}} \boldsymbol{Z} \boldsymbol{h}: \mathrm{st} \boldsymbol{X} \rightarrow \mathrm{st}^{3} \boldsymbol{Z}$. Using Theorem 3.94 ) twice, this implies (ALip) ${ }_{m+n+2}^{*}$ for $\mathrm{st}^{2} \boldsymbol{h}: \mathrm{st}^{3} \boldsymbol{X} \rightarrow \mathrm{st}^{3} \boldsymbol{Z}$.

Theorem 5.6. If approximate maps $\boldsymbol{f}: \boldsymbol{X} \rightarrow \boldsymbol{Y}$ and $\boldsymbol{g}: \boldsymbol{Y} \rightarrow \boldsymbol{Z}$ both have property (APS), then so does $\boldsymbol{h}:$ st $\boldsymbol{X} \rightarrow$ st $\boldsymbol{Z}$.

Proof. Let $k \in \mathbb{N}$, and let $\mathcal{W} \in \operatorname{Cov}\left(Z_{k}\right)$. Take $\mathcal{W}^{\prime} \in \operatorname{Cov}\left(Z_{k}\right)$ such that $\operatorname{st}^{2} \mathcal{W}^{\prime}<\mathcal{W}$. By (A3) and (APS) for $\boldsymbol{g}: \boldsymbol{Y} \rightarrow \boldsymbol{Z}$, there exists $k_{0}>k$ such that for each $k^{\prime}>k_{0}$,

$$
\mathcal{W}_{k^{\prime}}<r_{k k^{\prime}}^{-1} \mathcal{W}^{\prime}
$$

and there exists $k_{0}^{\prime}>k^{\prime}$ with the property that for each $k^{\prime \prime}>k_{0}^{\prime}$ there exists $j_{0}>g\left(k^{\prime}\right)$ such that for each $j^{\prime}>j_{0}$,

$$
r_{k k^{\prime \prime}}\left(Z_{k^{\prime \prime}}\right) \subseteq \operatorname{st}\left(r_{k k^{\prime}} g_{k^{\prime}} q_{g\left(k^{\prime}\right), j^{\prime}}\left(Y_{j^{\prime}}\right), \mathcal{W}^{\prime}\right) .
$$

Furthermore, if we fix $j^{\prime}>j_{0}$, by (APS) and (AM) for $\boldsymbol{f}: \boldsymbol{X} \rightarrow \boldsymbol{Y}$, there exists $j_{0}^{\prime}>j^{\prime}$ such that each $j^{\prime \prime}>j_{0}^{\prime}$ admits $i_{0}>f g\left(k^{\prime}\right), f\left(j^{\prime}\right)$ such that for each $i^{\prime}>i_{0}$,

$$
q_{g\left(k^{\prime}\right), j^{\prime \prime}}\left(Y_{j^{\prime \prime}}\right) \subseteq \operatorname{st}\left(q_{g\left(k^{\prime}\right), j^{\prime}} f_{j^{\prime}} p_{f\left(j^{\prime}\right), i^{\prime}}\left(X_{i^{\prime}}\right), g_{k^{\prime}}^{-1} r_{k k^{\prime}}^{-1} \mathcal{W}^{\prime}\right),
$$

and

$$
\left(q_{g\left(k^{\prime}\right), j^{\prime}} f_{j^{\prime}} p_{f\left(j^{\prime}\right), i^{\prime}}, f_{g\left(k^{\prime}\right)} p_{f g\left(k^{\prime}\right), i^{\prime}}\right)<\operatorname{st} \mathcal{V}_{g\left(k^{\prime}\right)} .
$$

Then by (5.24),

$$
r_{k k^{\prime}} g_{k^{\prime}} q_{g\left(k^{\prime}\right), j^{\prime \prime}}\left(Y_{j^{\prime \prime}}\right) \subseteq \operatorname{st}\left(r_{k k^{\prime}} g_{k^{\prime}} q_{g\left(k^{\prime}\right), j^{\prime}} f_{j^{\prime}} p_{f\left(j^{\prime}\right), i^{\prime}}\left(X_{i^{\prime}}\right), \mathcal{W}^{\prime}\right) .
$$

Since $\boldsymbol{g}: \boldsymbol{Y} \rightarrow \boldsymbol{Z}$ is uniform,

$$
\text { st } \mathcal{V}_{g\left(k^{\prime}\right)}<g_{k^{\prime}}^{-1} \text { st } \mathcal{W}_{k^{\prime}}
$$


By (5.26), (5.25), (5.27) and (5.22),

$$
r_{k k^{\prime}} g_{k^{\prime}} q_{g\left(k^{\prime}\right), j^{\prime \prime}}\left(Y_{j^{\prime \prime}}\right) \subseteq \operatorname{st}\left(r_{k k^{\prime}} g_{k^{\prime}} f_{g\left(k^{\prime}\right)} p_{f g\left(k^{\prime}\right), i^{\prime}}\left(X_{i^{\prime}}\right), \text { st } \mathcal{W}^{\prime}\right) .
$$

Since $j^{\prime \prime}>j_{0}$, by (5.23) and (5.28),

$$
\begin{aligned}
r_{k k^{\prime \prime}}\left(Z_{k^{\prime \prime}}\right) & \subseteq \operatorname{st}\left(r_{k k^{\prime}} g_{k^{\prime}} f_{g\left(k^{\prime}\right)} p_{f g\left(k^{\prime}\right) i^{\prime}}\left(X_{i^{\prime}}\right), \mathrm{st}^{2} \mathcal{W}^{\prime}\right) \\
& \subseteq \operatorname{st}\left(r_{k k^{\prime}} g_{k^{\prime}} f_{g\left(k^{\prime}\right)} p_{f g\left(k^{\prime}\right) i^{\prime}}\left(X_{i^{\prime}}\right), \mathcal{W}\right) .
\end{aligned}
$$

Thus, each $k^{\prime \prime}>k_{0}^{\prime}$ admits $i_{0}>f g\left(k^{\prime}\right)$ such that for each $i^{\prime}>i_{0}$,

$$
r_{k k^{\prime \prime}}\left(Z_{k^{\prime \prime}}\right) \subseteq \operatorname{st}\left(r_{k k^{\prime}} h_{k^{\prime}} p_{h\left(k^{\prime}\right) i^{\prime}}\left(X_{i^{\prime}}\right), \mathcal{W}\right),
$$

which verifies (APS) for $\boldsymbol{h}$.

Let $\boldsymbol{\varphi} \in \operatorname{LIP}(\boldsymbol{p}, \boldsymbol{q})$ and $\boldsymbol{\psi} \in \operatorname{LIP}(\boldsymbol{q}, \boldsymbol{r})$, where $\boldsymbol{p}=\left\{p_{i}\right\}: X \rightarrow \boldsymbol{X}=$ $\left\{X_{i}, \mathcal{U}_{i}, p_{i i^{\prime}}\right\}, \boldsymbol{q}=\left\{q_{j}\right\}: Y \rightarrow \boldsymbol{Y}=\left\{Y_{j}, \mathcal{V}_{j}, q_{j j^{\prime}}\right\}$ and $\boldsymbol{r}=\left\{r_{k}\right\}: Z \rightarrow$ $\boldsymbol{Z}=\left\{Z_{k}, \mathcal{W}_{k}, r_{k k^{\prime}}\right\}$ are admissible approximate resolutions. Let $\boldsymbol{\varphi}$ and $\boldsymbol{\psi}$ be represented by uniform approximate maps $\boldsymbol{f}: \mathrm{st}^{s} \boldsymbol{X} \rightarrow \mathrm{st}^{s} \boldsymbol{Y}$ with (ALip) ${ }_{m}^{*}$ and (APS) and $\boldsymbol{g}: \mathrm{st}^{t} \boldsymbol{Y} \rightarrow \mathrm{st}^{t} \boldsymbol{Z}$ with (ALip) ${ }_{n}^{*}$ and (APS) for some $m, n \in \mathbb{Z}$. By Theorem 5.3, taking the maximum of $s$ and $t$, we can assume $s=t$. If we let $h=f g$ and $h_{k}=g_{k} f_{g(k)}: X_{f g(k)} \rightarrow Z_{k}$, then by Theorem 5.4 $\boldsymbol{h}=\left\{h_{k}, h\right\}: \mathrm{st}^{s+1} \boldsymbol{X} \rightarrow \mathrm{st}^{s+1} \boldsymbol{Z}$ is a uniform approximate map which has property (APS), by Theorem 5.6. Note that st $\boldsymbol{f}: \mathrm{st}^{s+1} \boldsymbol{X} \rightarrow \mathrm{st}^{s+1} \boldsymbol{Y}$ has property (ALip) ${ }_{m+1}^{*}$ and $\mathrm{st}^{2} \boldsymbol{g}: \mathrm{st}^{s+2} \boldsymbol{Y} \rightarrow \mathrm{st}^{s+2} \boldsymbol{Z}$ has property (ALip) ${ }_{n+2}^{*}$ by Theorem 5.3. So Theorem 5.5 implies that $\mathrm{st}^{2} \boldsymbol{h}: \mathrm{st}^{s+3} \boldsymbol{X} \rightarrow \mathrm{st}^{s+3} \boldsymbol{Z}$ has property (ALip) ${ }_{m+n+5}^{*}$. Now define $\boldsymbol{\psi} \circ \boldsymbol{\varphi}$ as the morphism in $\operatorname{LIP}(\boldsymbol{p}, \boldsymbol{r})$ represented by $\mathrm{st}^{2} \boldsymbol{h}: \mathrm{st}^{s+3} \boldsymbol{X} \rightarrow \mathrm{st}^{s+3} \boldsymbol{Z}$. It is easy to see that the definition of $\boldsymbol{\psi} \circ \boldsymbol{\varphi}$ does not depend on the choice of the representative of $\boldsymbol{f}$ or $\boldsymbol{g}$. Let $1_{\boldsymbol{p}} \in \operatorname{LIP}(\boldsymbol{p}, \boldsymbol{p})$ be the morphisms represented by the identity approximate map $1_{\boldsymbol{X}}: \boldsymbol{X} \rightarrow \boldsymbol{X}$. Then it is easy to see $1_{\boldsymbol{q}} \circ \boldsymbol{\varphi}=\boldsymbol{\varphi}$ and $\varphi \circ 1_{p}=\boldsymbol{\varphi}$. Associativity of the composition also holds. Hence, we have

TheOREM 5.7. LIP is a category.

TheOREM 5.8. $\underline{\operatorname{Dim}}_{B}$ and $\overline{\operatorname{Dim}}_{B}$ are invariants in the category LIP.

Proof. Let $\boldsymbol{p}=\left\{p_{i}\right\}: X \rightarrow \boldsymbol{X}=\left\{X_{i}, \mathcal{U}_{i}, p_{i i^{\prime}}\right\}$ and $\boldsymbol{q}=\left\{q_{j}\right\}: Y \rightarrow$ $\boldsymbol{Y}=\left\{Y_{j}, \mathcal{V}_{j}, q_{j j^{\prime}}\right\}$ be admissible approximate resolutions. Suppose that $\boldsymbol{\varphi} \in$ $\operatorname{LIP}(\boldsymbol{p}, \boldsymbol{q})$ and $\boldsymbol{\psi} \in \operatorname{LIP}(\boldsymbol{q}, \boldsymbol{p})$ satisfy $\boldsymbol{\psi} \circ \boldsymbol{\varphi}=1_{\boldsymbol{p}}$ and $\boldsymbol{\varphi} \circ \boldsymbol{\psi}=1_{\boldsymbol{q}}$, and let $\boldsymbol{\varphi}$ and $\boldsymbol{\psi}$ be represented by $\boldsymbol{f}=\left\{f_{j}, f\right\} \in \mathrm{UALip}\left(\mathrm{st}^{m} \boldsymbol{X}, \mathrm{st}^{m} \boldsymbol{Y}\right)$ and $\boldsymbol{g}=\left\{g_{i}, g\right\} \in$ UALip $\left(\mathrm{st}^{m} \boldsymbol{Y}, \mathrm{st}^{m} \boldsymbol{X}\right)$, respectively, for some $m \geq 1$. Then $\boldsymbol{f}: \mathrm{st}^{m} \boldsymbol{X} \rightarrow \mathrm{st}^{m} \boldsymbol{Y}$ and $\boldsymbol{g}: \mathrm{st}^{m} \boldsymbol{Y} \rightarrow \mathrm{st}^{m} \boldsymbol{X}$ have properties (APS) and (ALip) ${ }_{l}^{*}$ for some $l \geq 0$. Thus by Theorems 3.9 and 4.3 ,

$$
\underline{\operatorname{dim}}_{B}\left(\mathrm{st}^{m} \boldsymbol{q}: Y \rightarrow \mathrm{st}^{m} \boldsymbol{Y}\right)=\underline{\operatorname{dim}}_{B}\left(\mathrm{st}^{m} \boldsymbol{p}: X \rightarrow \mathrm{st}^{m} \boldsymbol{X}\right),
$$

and

$$
\overline{\operatorname{dim}}_{B}\left(\mathrm{st}^{m} \boldsymbol{q}: Y \rightarrow \mathrm{st}^{m} \boldsymbol{Y}\right)=\overline{\operatorname{dim}}_{B}\left(\mathrm{st}^{m} \boldsymbol{p}: X \rightarrow \mathrm{st}^{m} \boldsymbol{X}\right),
$$


which implies

$$
\underline{\operatorname{Dim}}_{B}(\boldsymbol{q}: Y \rightarrow \boldsymbol{Y})=\underline{\operatorname{Dim}}_{B}(\boldsymbol{p}: X \rightarrow \boldsymbol{X}),
$$

and

as required.

$$
\overline{\operatorname{Dim}}_{B}(\boldsymbol{q}: Y \rightarrow \boldsymbol{Y})=\overline{\operatorname{Dim}}_{B}(\boldsymbol{p}: X \rightarrow \boldsymbol{X}),
$$

\section{Category Whose morphisms are BiLipschitz maps}

In this section we construct another category BILIP whose morphisms are based on those approximate maps which correspond to biLipschitz maps, so that this is another category where the box-counting dimension is invariant.

Remark 6.1. For each $m \in \mathbb{Z}$ and for each approximate map $\boldsymbol{f}: \boldsymbol{X} \rightarrow \boldsymbol{Y}$, in properties $(\mathrm{ALip})_{m},(\mathrm{ALip})^{m},(\mathrm{ALip})_{m}^{*},(\mathrm{ALip})_{*}^{m}$, replace "for each $i \in \mathbb{N}$ " by "there exists $N \in \mathbb{N}$ such that for each $i \geq N$ " and call the so obtained properties $\left.(\widehat{\mathrm{ALip}})_{m}, \widehat{(\mathrm{ALip})^{m}}, \widehat{(\widehat{\mathrm{ALip}})_{m}^{*}}, \widehat{\left(\mathrm{ALip}^{m}\right.}\right)_{*}^{m}$, respectively. In a similar way, for each $m, n \geq 0$, for each $f: X \rightarrow Y$, and for normal sequences $\mathbb{U}$ and $\mathbb{V}$ on $X$ and $Y$, respectively, define properties $\widehat{(\mathrm{M})}_{m, n}, \widehat{(\mathrm{N})}_{m, n}, \widehat{(\mathrm{M})}^{m, n}, \widehat{(\mathrm{N})}^{m, n}$ as properties $(\mathrm{M})_{m, n},(\mathrm{~N})_{m, n},(\mathrm{M})^{m, n},(\mathrm{~N})^{m, n}$ for $\Sigma^{N} \mathbb{U}$ and $\Sigma^{N} \mathbb{V}$ for some $N$, respectively. Then it is easy to see that all the results involving properties $(\text { ALip })_{m},(\text { ALip })^{m},(\text { ALip })_{m}^{*},(\text { ALip })_{*}^{m},(\mathrm{M})_{m, n},(\mathrm{~N})_{m, n},(\mathrm{M})^{m, n},(\mathrm{~N})^{m, n}$ also hold for properties $(\widehat{\mathrm{ALip}})_{m}, \widehat{(\widehat{\mathrm{ALip}})^{m}},(\widehat{\mathrm{ALip}})_{m}^{*}, \widehat{(\mathrm{ALip}}_{*}^{m}, \widehat{(\mathrm{M})_{m, n}}, \widehat{(\mathrm{N})}_{m, n}$, $\widehat{(\mathrm{M})}^{m, n}, \widehat{(\mathrm{N})}^{m, n}$.

Let the objects of BILIP be all addmissible approximate resolutions. We define the morphisms as follows: $\operatorname{Let} \operatorname{UABiLip}(\boldsymbol{X}, \boldsymbol{Y})$ denote the set of all uniform approximate maps with properties (APS), $(\widehat{\mathrm{ALip}})_{m}^{*}$ and $\left.\widehat{(\mathrm{ALip}}\right)_{*}^{n}$ for some $m, n \in \mathbb{Z}$.

TheOREM 6.2. Let $\boldsymbol{f}: \boldsymbol{X} \rightarrow \boldsymbol{Y}$ be an approximate map. Then if $\mathrm{st}^{2} \boldsymbol{f}$ : $\mathrm{st}^{2} \boldsymbol{X} \rightarrow \mathrm{st}^{2} \boldsymbol{Y}$ has property $\widehat{(\mathrm{ALip})_{*}^{m}}$ for some $m \in \mathbb{Z}$, then $\mathrm{st}^{3} \boldsymbol{f}: \mathrm{st}^{3} \boldsymbol{X} \rightarrow$

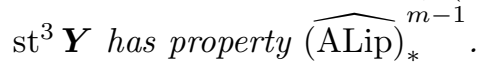

Proof. Assume $m \geq 1$ since the case $m \leq 0$ is similar. Take $N \in \mathbb{N}$ so that property $(\text { ALip })_{*}^{m}$ holds for each $i \geq N$, and let $i \geq N$. Then by $\widehat{(\mathrm{ALip}}_{*}^{m}$ for $\mathrm{st}^{2} \boldsymbol{f}: \mathrm{st}^{2} \boldsymbol{X} \rightarrow \mathrm{st}^{2} \boldsymbol{Y}$ and $(\mathrm{AM})$ for $\boldsymbol{f}: \boldsymbol{X} \rightarrow \boldsymbol{Y}$, there exists $i_{0}>i+m, f(i+1)$ such that for each $i^{\prime}>i_{0}$,

$$
p_{f(i), i^{\prime}}^{-1} f_{i}^{-1} \mathrm{st}^{2} \mathcal{V}_{i}<p_{i+m, i^{\prime}}^{-1} \mathrm{st}^{2} \mathcal{U}_{i+m}
$$

and

$$
\left(f_{i} p_{f(i), i^{\prime}}, q_{i, i+1} f_{i+1} p_{f(i+1), i^{\prime}}\right)<\operatorname{st} \mathcal{V}_{i}
$$


(6.2) and (6.1) imply

$$
\begin{aligned}
p_{f(i+1), i^{\prime}}^{-1} f_{i+1}^{-1} q_{i, i+1}^{-1} \mathrm{st} \mathcal{V}_{i} & <p_{f(i), i^{\prime}}^{-1} f_{i}^{-1} \mathrm{st}^{2} \mathcal{V}_{i}<p_{i+m, i^{\prime}}^{-1} \mathrm{st}^{2} \mathcal{U}_{i+m} \\
& <p_{i+m, i^{\prime}}^{-1} \mathrm{st}^{3} \mathcal{U}_{i+m}
\end{aligned}
$$

(U) implies

$$
\mathrm{st}^{3} \mathcal{V}_{i+1}<q_{i, i+1}^{-1} \text { st } \mathcal{V}_{i}
$$

By (6.3) and (6.4),

$$
p_{f(i+1), i^{\prime}}^{-1} f_{i+1}^{-1} \mathrm{st}^{3} \mathcal{V}_{i+1}<p_{i+m, i^{\prime}}^{-1} \mathrm{st}^{3} \mathcal{U}_{i+m},
$$

which means $(\widehat{\mathrm{ALip}})_{*}^{m-1}$ for $\mathrm{st}^{3} \boldsymbol{f}: \mathrm{st}^{3} \boldsymbol{X} \rightarrow \mathrm{st}^{3} \boldsymbol{Y}$.

Theorem 6.3. Let $\boldsymbol{p}=\left\{p_{i}\right\}: X \rightarrow \boldsymbol{X}=\left\{X_{i}, \mathcal{U}_{i}, p_{i i^{\prime}}\right\}$ and $\boldsymbol{q}=\left\{q_{j}\right\}$ : $Y \rightarrow \boldsymbol{Y}=\left\{Y_{j}, \mathcal{V}_{j}, q_{j j^{\prime}}\right\}$ be admissible approximate resolutions of $X$ and $Y$, respectively. For each approximate map $\boldsymbol{f}=\left\{f_{j}, f\right\}: \mathrm{st}^{3} \boldsymbol{X} \rightarrow \mathrm{st}^{3} \boldsymbol{Y}$ with properties (ALip) ${ }_{*}^{m+3}$ and (ALip) ${ }_{n}^{*}$ for some $m, n \in \mathbb{Z}$, there exists a uniform approximate map $\boldsymbol{f}^{\prime}=\left\{f_{j}^{\prime}, f^{\prime}\right\}:$ st $\boldsymbol{X} \rightarrow$ st $\boldsymbol{Y}$ with properties $\widehat{(\mathrm{ALip}}_{*}^{m}$ and $\widehat{(\mathrm{ALip}}_{n+1}^{*}$ which represents the same limit map $f: X \rightarrow Y$ as $\boldsymbol{f}$.

Proof. First assume that $m, n \geq 0$. For each $j$, let $f^{\prime}(j)$ be the smallest integer $i$ with the following properties:

1) $i>f(j)$;

2) $\left(p_{f(j), i^{\prime}} p_{i^{\prime} i^{\prime \prime}}, p_{f(j), i^{\prime \prime}}\right)<f_{j}^{-1} \mathcal{V}_{j}$ for $i^{\prime \prime}>i^{\prime} \geq i$;

3) $p_{f(j), i^{\prime}}^{-1} f_{j}^{-1} \mathrm{st}^{3} \mathcal{V}_{j}<p_{j+m+3, i^{\prime}}^{-1} \mathrm{st}^{3} \mathcal{U}_{j+m+3}$ for $i^{\prime} \geq i$;

4) $p_{j+n, i^{\prime}}^{-1}$ st $\mathcal{U}_{j+n}<p_{f(j), i^{\prime}}^{-1} f_{j}^{-1}$ st $\mathcal{V}_{j}$ for $i^{\prime} \geq i$; and

5) $i \geq f^{\prime}(i-1)$ if $j \geq 2$.

For each $j$, let $f_{j}^{\prime}: X_{f^{\prime}(j)} \rightarrow Y_{j}$ be defined as $f_{j}^{\prime}=f_{j} p_{f(j), f^{\prime}(j)}$.

Claim. $\boldsymbol{f}^{\prime}=\left\{f_{j}^{\prime}, f^{\prime}\right\}: \mathrm{st}^{4} \boldsymbol{X} \rightarrow \mathrm{st}^{4} \boldsymbol{Y}$ defines a uniform approximate map with properties $\widehat{(\mathrm{ALip}})_{*}^{m}$ and (ALip) ${ }_{n+1}^{*}$.

That $\boldsymbol{f}^{\prime}: \mathrm{st}^{4} \boldsymbol{X} \rightarrow \mathrm{st}^{4} \boldsymbol{Y}$ is a uniform approximate map with property $\left(\right.$ ALip) ${ }_{n+1}^{*}$ follows from the proof of Theorem 5.1. For each $j$, let $i_{0}=f^{\prime}(j)$, and let $i^{\prime}>i_{0}$. Then by 2 ),

$$
p_{f^{\prime}(j), i^{\prime}}^{-1} p_{f(j), f^{\prime}(j)}^{-1} f_{j}^{-1} \mathrm{st}^{2} \mathcal{V}_{j}<p_{f(j), i^{\prime}}^{-1} f_{j}^{-1} \mathrm{st}^{3} \mathcal{V}_{j}
$$

This together with 3 ) implies

$$
p_{f^{\prime}(j), i^{\prime}}^{-1} f_{j}^{\prime-1} \mathrm{st}^{2} \mathcal{V}_{j}<p_{j+m+3, i^{\prime}}^{-1} \mathrm{st}^{3} \mathcal{U}_{j+m+3}
$$

But

$$
\left(p_{j+m+2, i^{\prime}}, p_{j+m+2, j+m+3} p_{j+m+3, i^{\prime}}\right)<\mathcal{U}_{j+m+2},
$$

and so

$$
p_{j+m+3, i^{\prime}}^{-1} p_{j+m+2, j+m+3}^{-1} \mathrm{st}_{\mathcal{U}_{j+m+2}}<p_{j+m+2, i^{\prime}}^{-1} \mathrm{st}^{2} \mathcal{U}_{j+m+2} .
$$


By (U),

$$
\mathrm{st}^{3} \mathcal{U}_{j+m+3}<p_{j+m+2, j+m+3}^{-1} \mathrm{st}_{j+m+2} .
$$

By (6.5), (6.7) and (6.6),

$$
p_{f^{\prime}(j), i^{\prime}}^{-1} f_{j}^{\prime-1} \mathrm{st}^{2} \mathcal{V}_{j}<p_{j+m+2, i^{\prime}}^{-1} \mathrm{st}^{2} \mathcal{U}_{j+m+2}
$$

This means property (ALip) ${ }_{*}^{m+2}$ for $\boldsymbol{f}^{\prime}: \mathrm{st}^{2} \boldsymbol{X} \rightarrow \mathrm{st}^{2} \boldsymbol{Y}$. By Theorem 6.2, redefining $\boldsymbol{f}^{\prime}$ as $\mathrm{st}^{2} \boldsymbol{f}^{\prime}, \boldsymbol{f}^{\prime}: \mathrm{st}^{4} \boldsymbol{X} \rightarrow \mathrm{st}^{4} \boldsymbol{Y}$ has property $\left(\widehat{\mathrm{ALip}}_{*}{ }_{*}^{m}\right.$, which proves the claim.

For the case $m<0$ (resp., $n<0$ ), for each $j \in \mathbb{N}$, define $f^{\prime}(j)$ as $f(j)$, for $j \leq-m$ (resp., $j \leq-n$ ) and the smallest integer $i$ with properties 1$)-4$ ), for $j>-m$ (resp., $j>-n$ ). Then, by the same argument as above, we obtain a uniform approximate map $\boldsymbol{f}^{\prime}: \mathrm{st}^{4} \boldsymbol{X} \rightarrow \mathrm{st}^{4} \boldsymbol{Y}$ with properties $\widehat{(\mathrm{ALip}}_{*}^{m}$ and $\widehat{(\mathrm{ALip}}_{n+1}^{*}$.

By Theorems 5.3 and 6.2, there is a well-defined direct sequence

$$
\begin{aligned}
\text { UABiLip }\left(\mathrm{st}^{2} \boldsymbol{X}, \mathrm{st}^{2} \boldsymbol{Y}\right) \rightarrow & \text { UABiLip }\left(\mathrm{st}^{3} \boldsymbol{X}, \mathrm{st}^{3} \boldsymbol{Y}\right) \rightarrow \\
& \cdots \rightarrow \operatorname{UABiLip}\left(\mathrm{st}^{n} \boldsymbol{X}, \mathrm{st}^{n} \boldsymbol{Y}\right) \rightarrow \cdots .
\end{aligned}
$$

Let $\operatorname{UABiLip}^{*}(\boldsymbol{X}, \boldsymbol{Y})$ denote the direct limit of this sequence. For any admissible approximate resolutions $\boldsymbol{p}: X \rightarrow \boldsymbol{X}$ and $\boldsymbol{q}: Y \rightarrow \boldsymbol{Y}$, let the set $\operatorname{BILIP}(\boldsymbol{p}, \boldsymbol{q})$ of morphisms from $\boldsymbol{p}$ to $\boldsymbol{q}$ be the set UABiLip* $(\boldsymbol{X}, \boldsymbol{Y})$.

We define the composition similarly to the case of LIP: Let $\boldsymbol{f}=\left\{f_{j}, f\right\}$ : $\boldsymbol{X} \rightarrow \boldsymbol{Y}$ and $\boldsymbol{g}=\left\{g_{k}, g\right\}: \boldsymbol{Y} \rightarrow \boldsymbol{Z}$ be uniform approximate maps, where $\boldsymbol{p}=\left\{p_{i}\right\}: X \rightarrow \boldsymbol{X}=\left\{X_{i}, \mathcal{U}_{i}, p_{i i^{\prime}}\right\}, \boldsymbol{q}=\left\{q_{j}\right\}: Y \rightarrow \boldsymbol{Y}=\left\{Y_{j}, \mathcal{V}_{j}, q_{j j^{\prime}}\right\}$ and $\boldsymbol{r}=\left\{r_{k}\right\}: Z \rightarrow \boldsymbol{Z}=\left\{Z_{k}, \mathcal{W}_{k}, r_{k k^{\prime}}\right\}$ are admissible approximate resolutions. Define $h=f g: \mathbb{N} \rightarrow \mathbb{N}$ and for each $k$, define $h_{k}=g_{k} f_{g(k)}: X_{f g(k)} \rightarrow Y_{k}$. Then by Theorem $5.4, \boldsymbol{h}=\left\{h_{k}, h\right\}:$ st $\boldsymbol{X} \rightarrow$ st $\boldsymbol{Z}$ is a uniform approximate map.

THEOREM 6.4. If $\mathrm{st}^{3} \boldsymbol{f}: \mathrm{st}^{3} \boldsymbol{X} \rightarrow \mathrm{st}^{3} \boldsymbol{Y}$ has property (ALip) ${ }_{*}^{m}$ (resp., $\left(\widehat{\mathrm{ALip}}_{*}^{m}\right.$ ) and if $\mathrm{st}^{2} \boldsymbol{g}: \mathrm{st}^{2} \boldsymbol{Y} \rightarrow \mathrm{st}^{2} \boldsymbol{Z}$ has property (ALip) ${ }_{*}^{n}\left(\right.$ resp. , $\left(\widehat{\mathrm{ALip}}_{*}^{n}\right)$ for some $m, n \in \mathbb{Z}$, then $\boldsymbol{h}:$ st $\boldsymbol{X} \rightarrow$ st $\boldsymbol{Z}$ has property (ALip) ${ }_{*}^{m+n-2}$ (resp., $(\widehat{\mathrm{ALip}})_{*}^{m+n-2}$ ).

Proof. Assume $m, n \geq 1$ since the case for $m \leq 0$ or $n \leq 0$ is similar. Let $k \in \mathbb{N}$. By $(\mathrm{ALip})_{*}^{n}$ for $\mathrm{st}^{2} \boldsymbol{g}: \mathrm{st}^{2} \boldsymbol{Y} \rightarrow \mathrm{st}^{2} \boldsymbol{Z}$, there exists $j>k+n, g(k)$ such that

$$
q_{g(k), j}^{-1} g_{k}^{-1} \mathrm{st}^{2} \mathcal{W}_{k}<q_{k+n, j}^{-1} \mathrm{st}^{2} \mathcal{V}_{k+n}
$$

So,

$$
p_{f(j), i}^{-1} f_{j}^{-1} q_{g(k), j}^{-1} g_{k}^{-1} \mathrm{st}^{2} \mathcal{W}_{k}<p_{f(j), i}^{-1} f_{j}^{-1} q_{k+n, j}^{-1} \mathrm{st}^{2} \mathcal{V}_{k+n}
$$


By (AM) for $\boldsymbol{f}: \boldsymbol{X} \rightarrow \boldsymbol{Y}$ and (ALip) ${ }_{*}^{m}$ for $\mathrm{st}^{3} \boldsymbol{f}: \mathrm{st}^{3} \boldsymbol{X} \rightarrow \mathrm{st}^{3} \boldsymbol{Y}$, there is $i_{0}>f(k+n), f(j)(>g f(k))$ such that for each $i>i_{0}$,

$$
\begin{gathered}
\left(f_{k+n} p_{f(k+n), i}, q_{k+n, j} f_{j} p_{f(j), i}\right)<\operatorname{st} \mathcal{V}_{k+n}, \\
\left(f_{g(k)} p_{f g(k), i}, q_{g(k), j} f_{j} p_{f(j), i}\right)<\operatorname{st} \mathcal{V}_{g(k)},
\end{gathered}
$$

and

$$
p_{f(k+n), i}^{-1} f_{k+n}^{-1} \mathrm{st}^{3} \mathcal{V}_{k+n}<p_{k+n+m, i}^{-1} \mathrm{st}^{3} \mathcal{U}_{k+n+m}
$$

Fix $i>i_{0}$. Since $\boldsymbol{g}: \boldsymbol{Y} \rightarrow \boldsymbol{Z}$ is uniform, (6.10) implies

$$
\left(g_{k} f_{g(k)} p_{f g(k), i}, g_{k} q_{g(k), j} f_{j} p_{f(j), i}\right)<\mathrm{st} \mathcal{W}_{k},
$$

so

$$
p_{f g(k), i}^{-1} f_{g(k)}^{-1} g_{k}^{-1} \operatorname{st} \mathcal{W}_{k}<p_{f(j), i}^{-1} f_{j}^{-1} q_{g(k), j}^{-1} g_{k}^{-1} \mathrm{st}^{2} \mathcal{W}_{k}
$$

By (6.9),

$$
p_{f(j), i}^{-1} f_{j}^{-1} q_{k+n, j}^{-1} \mathrm{st}^{2} \mathcal{V}_{k+n}<p_{f(k+n), i}^{-1} f_{k+n}^{-1} \mathrm{st}^{3} \mathcal{V}_{k+n}
$$

By (6.12),(6.8), (6.13) and (6.11),

$$
p_{f g(k), i}^{-1} f_{g(k)}^{-1} g_{k}^{-1} \operatorname{st} \mathcal{W}_{k}<p_{k+n+m, i}^{-1} \mathrm{st}^{3} \mathcal{U}_{k+n+m} .
$$

But by (U),

$$
\begin{aligned}
p_{k+n+m, i}^{-1} \mathrm{st}^{3} \mathcal{U}_{k+n+m} & <p_{k+n+m, i}^{-1} \text { st } p_{k+n+m-1, k+n+m}^{-1} \mathcal{U}_{k+n+m-1} \\
& <p_{k+n+m, i}^{-1} p_{k+n+m-1, k+n+m}^{-1} \text { st } \mathcal{U}_{k+n+m-1}
\end{aligned}
$$

Since

$$
\left(p_{k+n+m-1, i}, p_{k+n+m-1, k+n+m} p_{k+n+m, i}\right)<\mathcal{U}_{k+n+m-1},
$$

$$
p_{k+n+m, i}^{-1} p_{k+n+m-1, k+n+m}^{-1} \operatorname{st}_{k+n+m-1}<p_{k+n+m-1, i}^{-1} \operatorname{st}^{2} \mathcal{U}_{k+n+m-1}
$$

By (6.15) and (6.16),

$$
p_{k+n+m, i}^{-1} \mathrm{st}^{3} \mathcal{U}_{k+n+m}<p_{k+n+m-1, i}^{-1} \mathrm{st}^{2} \mathcal{U}_{k+n+m-1} .
$$

By a similar argument,

$$
p_{k+n+m-1, i}^{-1} \mathrm{st}^{2} \mathcal{U}_{k+n+m-1}<p_{k+n+m-2, i}^{-1} \mathrm{st}_{\mathcal{U}_{k+n+m-2}} .
$$

By (6.14), (6.17) and (6.18), we have

$$
p_{f g(k), i}^{-1} f_{g(k)}^{-1} g_{k}^{-1} \text { st } \mathcal{W}_{k}<p_{k+n+m-2, i}^{-1} \text { st } \mathcal{U}_{k+n+m-2} \text { for } i>i_{0} .
$$

This means $(\mathrm{ALip})_{*}^{m+n-2}$ for $\boldsymbol{h}:$ st $\boldsymbol{X} \rightarrow$ st $\boldsymbol{Z}$. Similarly for $\widehat{(\mathrm{ALip}}_{*}^{m}$ and $\widehat{(\mathrm{ALip}})_{*}^{n}$. 
Let $\boldsymbol{\varphi} \in \operatorname{BILIP}(\boldsymbol{p}, \boldsymbol{q})$ and $\boldsymbol{\psi} \in \operatorname{BILIP}(\boldsymbol{q}, \boldsymbol{r})$ where $\boldsymbol{p}=\left\{p_{i}\right\}: X \rightarrow \boldsymbol{X}=$ $\left\{X_{i}, \mathcal{U}_{i}, p_{i i^{\prime}}\right\}, \boldsymbol{q}=\left\{q_{j}\right\}: Y \rightarrow \boldsymbol{Y}=\left\{Y_{j}, \mathcal{V}_{j}, q_{j j^{\prime}}\right\}$ and $\boldsymbol{r}=\left\{r_{k}\right\}: Z \rightarrow$ $\boldsymbol{Z}=\left\{Z_{k}, \mathcal{W}_{k}, r_{k k^{\prime}}\right\}$ are admissible approximate resolutions. Let $\boldsymbol{\varphi}$ and $\boldsymbol{\psi}$ be represented by uniform approximate maps $\boldsymbol{f}: \mathrm{st}^{s} \boldsymbol{X} \rightarrow \mathrm{st}^{s} \boldsymbol{Y}$ with properties $\left.(\widehat{\mathrm{ALip}})_{m}^{*}, \widehat{(\mathrm{ALip}}\right)_{*}^{k}$ and $(\mathrm{APS})$ and $\boldsymbol{g}: \mathrm{st}^{t} \boldsymbol{Y} \rightarrow \mathrm{st}^{t} \boldsymbol{Z}$ with properties $(\widehat{\mathrm{ALip}})_{n}^{*}$, $\widehat{(\mathrm{ALip}})_{*}^{l}$ and (APS) for some $m, n, k, l \in \mathbb{Z}$. By Theorems 5.3 and 6.2 , taking the maximum of $s$ and $t$, we can assume $s=t$. If we let $h=f g$ and $h_{k}=$ $g_{k} f_{g(k)}: X_{f g(k)} \rightarrow Z_{k}$, then $\boldsymbol{h}=\left\{h_{k}, h\right\}: \mathrm{st}^{s+1} \boldsymbol{X} \rightarrow \mathrm{st}^{s+1} \boldsymbol{Z}$ is a uniform approximate map by Theorem 5.4 and has property (APS), by Theorem 5.6. Similarly to the case of LIP, the approximate map st ${ }^{2} \boldsymbol{h}: \mathrm{st}^{s+3} \boldsymbol{X} \rightarrow \mathrm{st}^{s+3} \boldsymbol{Z}$ has property $\widehat{(\mathrm{ALip}})_{m+n+5}^{*}$. Also note that $\mathrm{st}^{3} \boldsymbol{f}: \mathrm{st}^{s+3} \boldsymbol{X} \rightarrow \mathrm{st}^{s+3} \boldsymbol{Y}$ has

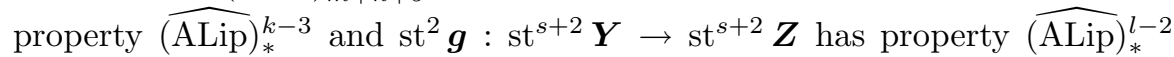
by Theorem 6.2. So Theorem 6.4 implies that $\boldsymbol{h}: \mathrm{st}^{s+1} \boldsymbol{X} \rightarrow \mathrm{st}^{s+1} \boldsymbol{Z}$ has property $\widehat{(\mathrm{ALip}})_{*}^{k+l-7}$, and hence by Theorem 6.2 again, $\mathrm{st}^{2} \boldsymbol{h}: \mathrm{st}^{s+3} \boldsymbol{X} \rightarrow$ $\mathrm{st}^{s+3} \boldsymbol{Z}$ has property $\left.\widehat{(\mathrm{ALip}}\right)_{*}^{k+l-9}$. Now define $\boldsymbol{\psi} \circ \boldsymbol{\varphi}$ as the morphism in $\operatorname{BILIP}(\boldsymbol{p}, \boldsymbol{r})$ represented by $\mathrm{st}^{2} \boldsymbol{h}: \mathrm{st}^{s+3} \boldsymbol{X} \rightarrow \mathrm{st}^{s+3} \boldsymbol{Z}$. It is easy to see that the definition of $\psi \circ \varphi$ does not depend on the choice of the representative $\boldsymbol{f}$ or $\boldsymbol{g}$. Let $1_{\boldsymbol{p}} \in \operatorname{BILIP}(\boldsymbol{p}, \boldsymbol{p})$ be the morphism represented by the identity approximate map $1_{\mathrm{st}^{2} \boldsymbol{X}}: \mathrm{st}^{2} \boldsymbol{X} \rightarrow \mathrm{st}^{2} \boldsymbol{X}$. Then it is easy to see $1_{q} \circ \boldsymbol{\varphi}=\boldsymbol{\varphi}$ and $\varphi \circ 1_{p}=\varphi$. Associativity of the composition also holds. Hence, we have

THEOREM 6.5. BILIP is a category.

and

TheOREM 6.6. $\underline{\operatorname{Dim}}_{B}$ and $\overline{\operatorname{Dim}}_{B}$ are invariants in the category BILIP.

Proof. This follows from Corollary 4.5.

\section{REFERENCES}

[1] P. Alexandroff and P. Urysohn, Une condition nécessaire et suffisante pour qu'une classe (L) soit une classe (B), C. R. Acad. Sci. Paris Sér. A-B 177 (1923), 1274-1276.

[2] S. Mardešić, Mappings of inverse systems, Glas. Mat. 18 (1963), 241-254.

[3] S. Mardešić and L. Rubin, Approximate inverse systems of compacta and covering dimension, Pacific J. Math. 138 (1989), 129-144.

[4] S. Mardešić and J. Segal, $\mathcal{P}$-like continua and approximate inverse limits, Math. Japonica 33(6) (1988), 895-908.

[5] S. Mardešić and T. Watanabe, Approximate resolutions of spaces and maps, Glas. Mat. 24 (1989), 587-637.

[6] J. Mioduszewski, Mappings of inverse limits, Colloquium Math. 10 (1963), 39-44.

[7] T. Miyata and T. Watanabe, Approximate resolutions of uniform spaces, Topology Appl. 113 (1-3) (2001), 211-241.

[8] T. Miyata and T. Watanabe, Approximate resolutions and Lipschitz functions, Topology Appl. 122 (2002), 353-375.

[9] T. Miyata and T. Watanabe, Approximate resolutions and box-counting dimension, Topology Appl. (to appear). 
[10] T. Miyata and T. Watanabe, Approximate resolutions and the fractal category, Glas. Mat. (to appear).

[11] K. Nagami, Dimension Theory, Academic Press, New York, 1970.

[12] J. Segal and T. Watanabe, Cosmic approximate limits and fixed points, Trans. Amer. Math. Soc. 333 (1992), 1-61.

[13] T. Watanabe, Approximate expansions of maps into inverse systems, in: Geometric and Algebraic Topology, Banach Center Publ. 18 (1986), 363-370.

[14] T. Watanabe, Approximative shape $I$ - IV, Tsukuba J. Math. 11 (1987), 17-59; 11 (1987), 303-339; 12 (1988), 1-41; 12 (1988), 273-319.

[15] T. Watanabe, Approximate resolutions and covering dimension, Topology Appl. 38 (1991), 147-154.

T. Miyata

Division of Mathematics and Informatics

Faculty of Human Development, Kobe University

Nada-Ku, 3-11 Tsurukabuto, Kobe, 657-8501

JAPAN

E-mail: tmiyata@kobe-u.ac.jp

T. Watanabe

Department of Mathematics and Information Sciences

Faculty of Education, Yamaguchi University

Yamaguchi-City, 753-8513

JAPAN

E-mail: tadashi@po.yb.cc.yamaguchi-u.ac.jp

Received: 21.11.2001. 